\title{
Exploratory multivariate analysis for empirical information affected by uncertainty and modeled in a fuzzy manner: a review
}

\author{
Pierpaolo D'Urso ${ }^{1}$
}

Received: 18 September 2016 / Accepted: 25 January 2017 / Published online: 24 March 2017

(c) Springer International Publishing Switzerland 2017

\begin{abstract}
In the last few decades, there has been an increase in the interest of the scientific community for multivariate statistical techniques of data analysis in which the data are affected by uncertainty, imprecision, or vagueness. In this context, following a fuzzy formalization, several contributions and developments have been offered in various fields of the multivariate analysis. In this paper-to show the advantages of the fuzzy approach in providing a deeper and more comprehensive insight into the management of uncertainty in this branch of Statistics-we present an overview of the developments in the exploratory multivariate analysis of imprecise data. In particular, we give an outline of these contributions within an overall framework of the general fuzzy approach to multivariate statistical analysis and review the principal exploratory multivariate methods for imprecise data proposed in the literature, i.e., cluster analysis, self-organizing maps, regression analysis, principal component analysis, multidimensional scaling, and other exploratory statistical approaches. Finally, we point out certain potentially fruitful lines of research that could enrich the future developments in this interesting and promising research area of Statistical Reasoning. All in all, the main purpose of this paper is to involve the Granular Computing scientific community and to stimulate and focus its interest on these statistical fields.
\end{abstract}

Pierpaolo D'Urso

pierpaolo.durso@uniroma1.it

1 Department of Social Sciences and Economics, SapienzaUniversity of Rome, Rome, Italy
Keywords Statistical reasoning · Information · Uncertainty and imprecision · Granularity · Information granules - Exploratory multivariate statistical analysis . Fuzzy data $\cdot$ Metrics for fuzzy data $\cdot$ Cluster analysis . Regression analysis $\cdot$ Self-organizing maps $\cdot$ Principal component analysis $\cdot$ Multidimensional scaling · Correspondence analysis - Clusterwise regression analysis . Classification and regression trees · Three-way analysis

\section{Introduction}

In Statistical Reasoning, the basic ingredients of a statistical analysis are data and models. Both share an informational nature, which can be clearly sized in the knowledge discovery process leading to the acquisition of an informational gain. These informational objects are often uncertain, imprecise, or vaguely defined. Fuzzy sets theory (Zadeh 1965) may take into account this uncertainty and imprecision, providing us with a means for dealing with fuzzy informational objects in statistical analysis. Early contributions deal mainly with cluster analysis and regression. New developments have been taking place in the last decades, in the context of a more systematic approach to fuzzy statistical methods (Coppi 2003). New techniques of analysis have been proposed in the literature, extending the domain of fuzzy exploratory multivariate analysis to cluster analysis, regression analysis, principal component analysis, multidimensional scaling, self-organizing maps, clusterwise regression analysis, three-way analysis, and recently regression trees.

The aim of this paper is to give an outline of these developments within an organic framework acting as the basis for the general fuzzy approach to multivariate statistical analysis. In this connection-focusing our attention on the 
exploratory multivariate analysis of imprecise empirical information and considering a fuzzy approach to manage the uncertainty affecting the information (data and models) - we present a review of the exploratory multivariate methods proposed in the literature, i.e., cluster analysis, self-organizing maps, regression analysis, principal component analysis, multidimensional scaling, and other exploratory multivariate methods.

Overall, the main purpose of this paper is to involve the Granular Computing scientific community and to stimulate and focus its interest in these statistical fields.

The paper is structured as follows: In Sect. 2, we analyze the informational paradigm and the uncertainty connected to information. In Sect. 3, we focus our attention on empirical information affected by uncertainty formalized in a fuzzy manner, i.e., fuzzy data. In Sect. 4, we present an organic review of exploratory multivariate methods for fuzzy data, i.e., cluster analysis (Sect. 4.1), self-organizing maps (Sect. 4.2), regression analysis (Sect. 4.3), principal component analysis (Sect. 4.4), multidimensional scaling (Sect. 4.5), and other exploratory multivariate methods for fuzzy data (Sect. 4.6). In conclusion, in Sect. 5, we point out some potentially fruitful lines of research that could lead to future developments in this interesting and promising research area of Statistical Reasoning.

\section{Information and uncertainty}

A corpus of knowledge is a set of information elements. Each information element is represented by the following quadruple: (attribute, object, value, confidence), where attribute is the function which maps an object into a value, in the framework of a reference universe of elements; value is the predicate of the object, associated to a subset of reference universe; confidence is the indication of the reliability of the information element (Coppi 2003).

In the real world, an element of information is generally affected by imprecision (with respect to value) and uncertainty (expressed through the notion of confidence). Both imprecision and uncertainty can be measured. When the measure of imprecision and/or uncertainty is different from zero, we have imperfect information (Coppi 2003).

In the reasoning process applied to a corpus of knowledge, a system of rules is adopted. This allows us to reach conclusions which, when the initial information is imperfect, are themselves characterized by imprecision and uncertainty. When the phenomena and the situations under investigation are complex, the following Incompatibility Principle (Zadeh 1973) should be taken into account: "As the complexity of a system increases, our ability to make precise and yet significant statements about its behavior diminishes until a threshold is reached beyond which precision and significance (or relevance) become almost mutually exclusive characteristics" (Zadeh 1973). This principle justifies the development and application of logics allowing the utilization of imprecise information, for drawing relevant conclusions when faced with complex contexts (Coppi 2003).

In Statistical Reasoning, the statistical method represents a special category of knowledge acquisition, since it defines a class of procedures referring to a specific type of information elements and to the associated processing techniques. This class is characterized by two essential entities: the empirical data and the models for data analysis, which in turn include both the theoretical assumptions and the algorithms for applying them to the data. The empirical data are special information elements, in which the attribute is represented by a statistical variable and the object by a statistical unit. The model for the data analysis can be considered as an information ingredient of a theoretical type, referring to abstract entities, such as variables, parameters, potential data, functional forms, and so on (Coppi 2003).

Thus, a statistical analysis can be described as a process of knowledge acquisition which, starting from an empirical and theoretical initial information, applies computational procedures (algorithms), thus acquiring additional information (information gain) having a cognitive and/or operational character (Coppi 2002).

Let us denote by $\left(\mathrm{I}_{\mathrm{E}}, \mathrm{I}_{\mathrm{T}}\right)$ the pair empirical information $\mathrm{I}_{\mathrm{E}}$ (data) and theoretical information $\mathrm{I}_{\mathrm{T}}$ (real world theories, mathematical formalizations, general and statistical assumptions, and so on). Both $\mathrm{I}_{\mathrm{E}}$ and $\mathrm{I}_{\mathrm{T}}$, to the extent to which they are informational entities (corpus of knowledge), are constituted by information elements which can be imprecise and uncertain. Generally, in real world applications, the pair $\left(\mathrm{I}_{\mathrm{E}}, \mathrm{I}_{\mathrm{T}}\right)$ represents imperfect information. A way to account for the imperfection of the information utilized in statistical analysis is to manage it using the fuzzy sets theory introduced by Zadeh (1965). This implies assuming that the pair $\left(\mathrm{I}_{\mathrm{E}}, \mathrm{I}_{\mathrm{T}}\right)$ has a fuzzy nature, i.e., we can have the following cases: $\mathrm{I}_{\mathrm{E}}$ is fuzzy, $\mathrm{I}_{\mathrm{T}}$ is fuzzy, both $\mathrm{I}_{\mathrm{E}}$ and $\mathrm{I}_{\mathrm{T}}$ are fuzzy (see, e.g., Coppi et al. 2006b; Coppi 2008).

In this paper, we will follow an exploratory approach, focusing our attention multivariate analysis in which theoretical methods $\left(\mathrm{I}_{\mathrm{T}}\right)$ and empirical data $\left(\mathrm{I}_{\mathrm{E}}\right)$ are, respectively, crisp/fuzzy and fuzzy.

\section{Empirical information and uncertainty: imprecise data}

In Statistical Reasoning, the uncertainty affecting empirical information $\left(\mathrm{I}_{\mathrm{E}}\right)$ may stem from various sources (Coppi and D'Urso 2012): 
- imprecision in measuring the empirical phenomenon represented by statistical variables;

- vagueness of statistical variables when these are expressed in linguistic terms;

- partial or total ignorance concerning the values taken by statistical variables on specific observational instances;

- "granularity" of statistical variables, with reference to the way it is defined and used in the analysis (e.g., the age of a person may be described in terms of 5-year intervals, or just as "young," "middle age," "old"; to each of these "granulations" there is associated a different amount of uncertainty) (see, e.g., Zadeh 2005; Pedrycz 2013).

These kinds of uncertainty can be modeled in a fuzzy manner (Zadeh 1965). In the following, we present a review of works in which the empirical information is analyzed by means of fuzzy tools in a multivariate statistical framework.

\subsection{Fuzzy data: mathematical and graphical representation}

In exploratory multivariate analysis, following the fuzzy sets theory (Zadeh 1965), imprecise empirical information (fuzzy data) can be formalized by considering a general class of fuzzy data, i.e., the so-called LR fuzzy data (Dubois and Prade 1988; Zimmermann 2001). These data can be stored in a (fuzzy) data matrix, i.e., LR fuzzy data matrix, ( $n$ observation units $\times p$ (fuzzy) variables) defined as follows:

$\tilde{\mathbf{X}} \equiv\left\{\tilde{x}_{i j}=\left(c_{1 i j}, c_{2 i j}, l_{i j}, r_{i j}\right)_{L R}: i=1, \ldots, n ; j=1, \ldots, p\right\}$

where $\tilde{x}_{i j}=\left(c_{1 i j}, c_{2 i j}, l_{i j}, r_{i j}\right)_{L R}$ represents the $L R$ fuzzy variable $j$ observed on the $i$-th unit, $c_{1 i j}$ and $c_{2 i j}$ indicate the left and right center, respectively, (the interval $\left[c_{1 i j}, c_{2 i j}\right]$ is usually referred to as the "core" of $\tilde{x}_{i j}$ ) and $l_{i j}$ and $r_{i j}$ the left and right spread, respectively, with the following membership function:

$\mu_{\tilde{x}_{i j}}\left(u_{i j}\right)=\left\{\begin{array}{lc}L\left(\frac{c_{1 i j}-u_{i j}}{l_{i j}}\right) & u_{i j} \leq c_{1 i j}\left(l_{i j}>0\right), \\ 1 & c_{1 i j} \leq u_{i j} \leq c_{2 i j}, \\ R\left(\frac{u_{i j}-c_{2 i j}}{r_{i j}}\right) & u_{i j} \geq c_{2 i j}\left(r_{i j}>0\right),\end{array}\right.$

where $L\left(z_{i j}\right)$ (and $\left.R\left(z_{i j}\right)\right)\left(z_{i j}\right.$ is a generic variable, e.g., $\left.z_{i j}=\left(c_{1 i j}-u_{i j}\right) / l_{i j}\right)$ is a decreasing 'shape' function from $\mathfrak{R}^{+}$to [0,1] with $L(0)=1 ; L\left(z_{i j}\right)<1$ for all $z_{i j}>0, \forall i, j$; $L\left(z_{i j}\right)>0$ for all $z_{i j}<1, \forall i, j ; L(1)=0$ (or $L\left(z_{i j}\right)>0$ for all $z_{i j}, \forall i, j$, and $L(+\infty)=0$ ) (Zimmermann 2001). The fuzzy number $\tilde{x}_{i j}=\left(c_{1 i j}, c_{2 i j}, l_{i j}, r_{i j}\right)_{L R}, i=1, \ldots, n ; j=1, \ldots, p$ consists of an interval which runs from $c_{1 i j}-l_{i j}$ to $c_{2 i j}+r_{i j}$ and the membership functions give differential weights to the values in the interval, respectively, to the left and to the right of the left and right "centers."

The most common LR fuzzy datum is the trapezoidal one (with trapezoidal membership function). In particular, for a LR fuzzy number $\tilde{x}_{i j}$, if $L$ and $R$ are of the form:

$L(z)=R(z)=\left\{\begin{array}{lc}1-z^{\alpha} & 0 \leq z \leq 1 \\ 0 & \text { otherwise, }\end{array}\right.$

with $\alpha=1$ then $\mathbf{X}=\left\{\tilde{x}_{i j}: i=1 \ldots, n ; j=1, \ldots, p\right\}$ is a trapezoidal fuzzy data matrix whose elements have the following membership functions:

$\tilde{\mu}_{x_{i j}}\left(u_{i j}\right)=\left\{\begin{array}{lc}1-\frac{c_{1 i j}-c_{i j}}{l_{i j}} & u_{i j} \leq c_{1 i j}\left(l_{i j}>0\right) \\ 1 & c_{1 i j} \leq u_{i j} \leq m_{2 i j} \\ 1-\frac{c_{i j}-c_{2 i j}}{r_{i j}} & u_{i j} \geq m_{2 i j}\left(r_{i j}>0\right) .\end{array}\right.$

When $c_{1 i j}=m_{2 i j}$, we obtain a particular type of LR fuzzy number, denoted as $\tilde{x}_{i j}=\left(c_{i j}, l_{i j}, r_{i j}\right)_{L R}$, where $m_{i j}$ indicates the center, $i=1, \ldots, n ; j=1, \ldots, p$, determining the following particular case of LR fuzzy data matrix:

$\mathbf{X} \equiv\left\{\tilde{x}_{i j}=\left(c_{i j}, l_{i j}, r_{i j}\right)_{L R}: i=1, \ldots, n ; j=1, \ldots, p\right\}$.

Particular cases of LR fuzzy data are the triangular, parabolic, and square root ones (when $L$ and $R$ are shown in Eq. (3) with $\alpha=1, \alpha=2$, and $\alpha=1 / 2$, respectively). Each case takes into account a different level of fuzziness around the centers of the fuzzy numbers. Specifically, the square root case denotes a low level of fuzziness, the triangular case a medium level, and the parabolic case a high level.

An alternative formalization of trapezoidal fuzzy data has been proposed by Hathaway et al. (1996) and Yang and Ko (1996).

Examples of membership functions generated by the family of membership function shown in Eq. (4) are illustrated in Fig. 1. In particular, in Fig. 1, we show the process of transformation from trapezoidal fuzzy data to triangular fuzzy data and uniform fuzzy data and then to crisp (nonfuzzy) data (D’Urso and De Giovanni 2014).

\subsection{Fuzzy data: elicitation and specification of the membership functions}

Two very important topics, connected with the representation of certain terms of natural language by means of fuzzy data, are the elicitation and specification of the membership functions. 


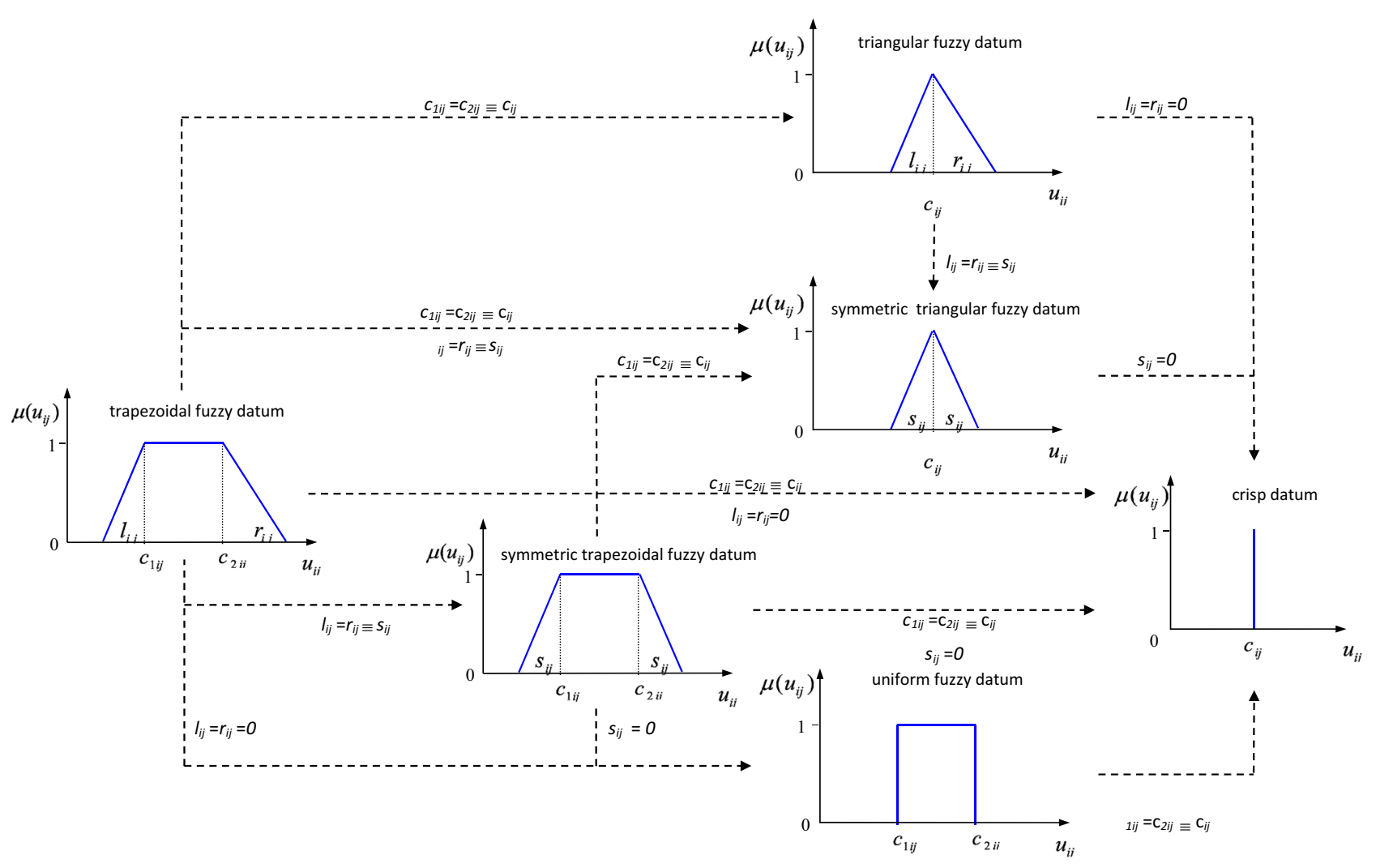

Fig. 1 Trapezoidal fuzzy data and their transformations. (source D’Urso and De Giovanni 2014)

- Elicitation of the membership functions As remarked by Coppi et al. (2006a) "as for the subjectivistic approach to probability, also the choice of the membership functions is subjective. In general, these are determined by experts in the problem area. In fact, the membership functions are context-sensitive. Furthermore, the functions are not determined in an arbitrary way, but are based on a sound psychological/linguistic foundation. It follows that the choice of the membership function should be made in such a way that a function captures the approximate reasoning of the person involved. In this respect, the elicitation of a membership function requires a deep psychological understanding."

- Specification of the membership functions In the statistical analysis of fuzzy multivariate data, attention must be paid to the specification of the membership functions when we deal simultaneously with a set of $p$ variables. In this case, we can consider two approaches: the conjunctive approach and the disjunctive approach (Coppi 2003). In the conjunctive approach, we take into account the fuzzy relationship defined on the Cartesian product of the reference universes of the $p$ variables. Within this perspective, we can distinguish non-interactive and interactive variables. From a statistical point of view, the adoption of the conjunctive approach to the multidi- mensional fuzzy variables involves a specific interest in studying the fuzzy relationship treated as a "variable" in itself, which can be observed in $n$ objects. Conversely, in the disjunctive approach, we are not interested in studying a fuzzy variable which constitutes the resultant of the $p$ original variables. Instead, our interest focuses upon the set of the $p$ "juxtaposed" variables, observed as a whole in the group of $n$ objects. In this case, we have $p$ membership functions and the investigation of the links among the $p$ fuzzy variables is carried out directly on the matrix of fuzzy data concerning the $n p$ variate observations (Coppi 2003; D'Urso 2007). Without loss in generality, let us consider the bi-dimensional case; furthermore, let be assumed that a unidimensional fuzzy variable is represented by a (symmetrical) triangular membership function and a bi-dimensional fuzzy variable is represented by two (symmetrical) triangular membership function (disjunctive approach) or a conical membership function (conjunctive approach). An example of geometrical representation of the considered membership functions based on the disjunctive and conjunctive approach is shown in Fig. 2. An analytical representation of two triangular fuzzy variables with (symmetrical) triangular membership functions (disjunctive approach) (Coppi 2003; D'Urso 2007) can be 
Fig. 2 Examples of membership functions based on the disjunctive and conjunctive approach. (source Celminš 1987, 1991)

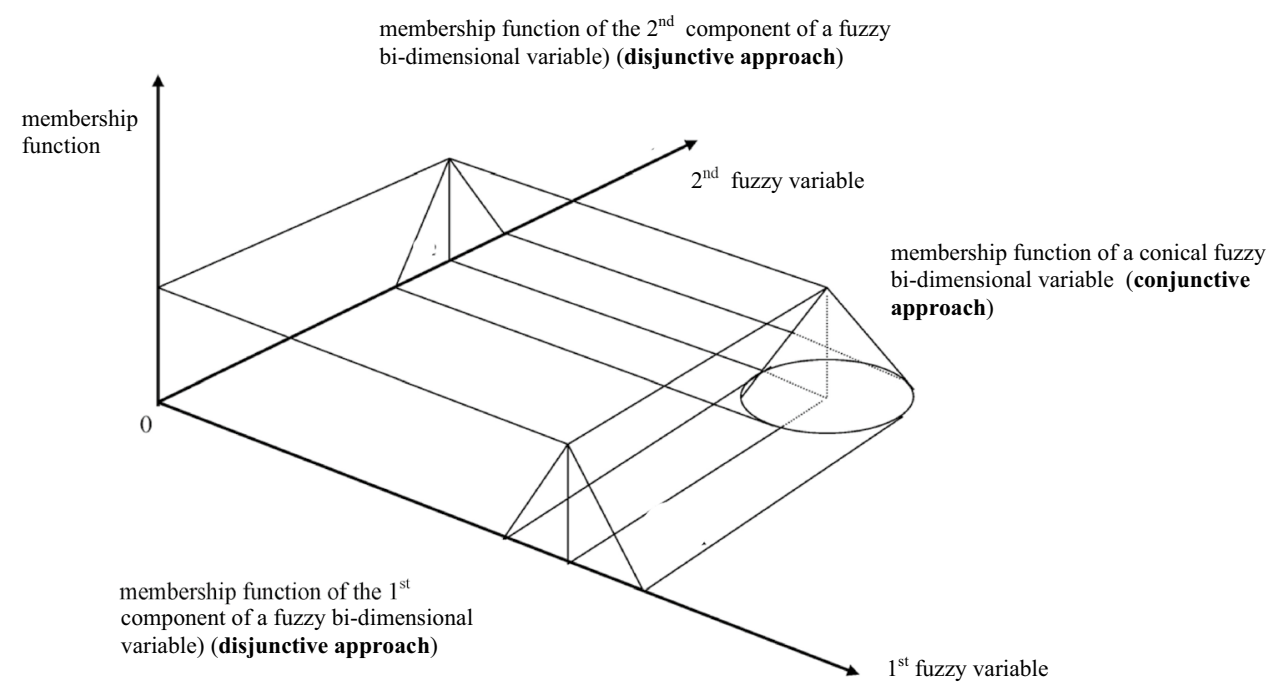

easy obtained by (4) fixing $c_{1 i j}=\mathrm{c}_{2 i j}, 1_{i j}=\mathrm{r}_{i j}$ and $j=1,2$. For an analytical formalization of a conical fuzzy variable with conical membership function (conjunctive approach), see Celminš (1987, 1991).

\subsection{Fuzzy data: fuzzy representation of linguistic terms, ordinal scales, and categorical data}

In the literature, there are different tools for treating in a fuzzy manner the imprecise empirical information represented by linguistic terms, ordinal scales, categorical data.

In particular, we have the following approaches:

- Fuzzy linguistic scales These scales are frequently considered for different goals as an a posteriori tool to encode data from a discrete (often a Likert) scale by means of fuzzy numbers (see, for instance, Zadeh 1975a, b, c; Tong and Bonissone 1980; Pedrycz 1989; Herrera et al. 1998, 2008; Lalla et al. 2008; Li 2013; Akdag et al. 2014; Estrella et al. 2014; Massanet et al. 2014; Tejeda-Lorente et al. 2014, 2015; Villacorta et al. 2014; Wang et al. 2014; Garcia-Galán et al. 2015; Liu et al. 2015a; Tavana et al. 2015).

- Fuzzy rating scale This scale has been introduced by Hesketh et al. (1988). See also, among others, Hesketh and Hesketh (1994), Matsui and Takeya (1994), Takemura (1999, 2007, 2012), Yamashita (2006), Hesketh et al. (2011) and de la Rosa de Saa et al. (2015), Lubiano et al. (2016a, b) for some developments and applications. This kind of scale is considered as an a priori tool to directly assess fuzzy values and integrating the continuous nature and free assessment of the visual analogue scales with the ability to cope with imprecision of the fuzzy linguistic ones. This kind of scale inherits the advantages of the visual analogue scale (this scale can be utilized in questionnaires, and in accordance with which respondents specify their rating by indicating a position along a continuous line between two endpoints, i.e., by choosing a point within a given bounded interval). In particular, in a fuzzy rating scale along a continuous line between two end-points: (1) a respondent selects or draws a 'representative position/interval' of the respondent rating (i.e., the set of points which she/he considers to be fully compatible with such a rating); (2) and the respondent also indicates 'latitudes of acceptance' on either side by determining the highest and lowest possible positions for the respondent rating (i.e., the set of points she/he considers to be compatible to some extent with such a rating). In this way, the fuzzy rating scale takes advantage of the synergies existing between the visual analogue and the fuzzy linguistic scales (de la Rosa de Saa et al. 2015). See de la Rosa de Saa et al. (2015) for a detailed discussion on the advantages of this kind of scale and for theoretical, comparative, and applicative aspects.

For a deep and interesting discussion on the different approaches, see de la Rosa de Saa et al. (2015) and Lubiano et al. $(2016 a, b)$.

\subsection{Fuzzy data: metrics}

In the literature, several proximity measures-dissimilarity, similarity, and distance measures-have been proposed for comparing pairs of objects in which imprecise (i.e., fuzzy) variables have been observed (D'Urso 2007). Some of these proximity measures are defined by taking into account the membership functions of the fuzzy data. 
These distances can be classified according to the following approaches (D’Urso et al. 2014; Bloch 1999; Zwick et al. 1987):

- Functional approach Based on the comparison of the membership functions by means of Minkowski and Canberra distance measures (Lowen and Peeters 1998; Pappis and Karacapilidis 1993).

- Information theoretic approach Based on the definition of fuzzy entropy (De Luca and Termini 1972).

- Set theoretic approach Based on the concepts of fuzzy union and intersection (Chen et al. 1995; Pappis and Karacapilidis 1993; Wang 1997; Zwick et al. 1987).

- Weighting system approach Based on the comparison of the fuzzy data by using directly the empirical information represented by the centers and the spreads of the fuzzy data and by adopting suitable weighting systems that somehow capture the information connected to the shape of the membership functions (see, e.g., Coppi et al. 2012; D’Urso and Giordani 2006a; Yang et al. 2004; Yang and Ko 1996; Yang and Liu 1999).

In particular, focusing on the last theoretical approach, Coppi et al. (2012) proposed a dissimilarity measure for LR fuzzy data that represent a generalization of the dissimilarity for symmetrical fuzzy data suggested by D'Urso and Giordani (2006a). By means of these measures, the dissimilarity between each pair of objects is computed by comparing the fuzzy data observed on each object, i.e., by considering, separately, the (squared) Euclidean distances for the centers and the spreads of the fuzzy data and using a suitable weighting system for such distance components. The weights can be chosen subjectively a priori by using suitably external or subjective information (external weighting system) or can be computed objectively within a suitable data analysis procedure (internal weighting system). In their methods, D'Urso and Giordani (2006a) and Coppi et al. (2012) opted for an internal weighting system. These dissimilarity measures based on the internal weighting system have been utilized in different exploratory multivariate methods (see, for instance, D'Urso and De Giovanni 2014; D'Urso et al. 2014). Yang and Ko (1996) suggested a dissimilarity measure for univariate fuzzy data, in which the selection of the weights is external. The Yang-Ko's distance has been used in Cappelli et al. (2013). D'Urso and De Giovanni (2014) and D'Urso et al. (2014) used in their methods a multivariate version of dissimilarity measure proposed by the Yang and Ko. For more details, see D'Urso et al. (2014).

Following a conjunctive approach for the specification of the membership functions, Yang and Liu (1999) proposed a suitable distance measure for conical fuzzy data.

\subsection{Fuzzy data: definitions in an inferential framework}

In the literature, we find approaches in which uncertainty is modeled using simultaneously the random (stochastic) approach and the fuzzy one by means of the concept of Fuzzy Random Variables (FRVs). From a theoretical viewpoint, this implies constructing appropriate probability measures of fuzzy sets. Various proposals have been made, in the last decades, based on this theoretical perspective. One of the most significant proposals is the notion of FRV used by Puri and Ralescu (1986) and Klement et al. (1986). Another definition for a fuzzy-valued random variable which is mathematically equivalent, albeit conceptually different and which can only be stated for the univariate case, is the one proposed by Kwakernaak $(1978,1979)$ and Kruse and Meyer (1987).

\section{Theoretical information and uncertainty: exploratory multivariate analysis of imprecise data}

In the following, we present a systematic review of the principal exploratory multivariate methods for imprecise data proposed in the literature. In particular, we review the following exploratory multivariate approaches based on a fuzzy framework:

- cluster analysis (Sect. 4.1),

- self-organizing maps (Sect. 4.2),

- regression analysis (Sect. 4.3),

- principal component analysis (Sect. 4.4),

- multidimensional scaling (Sect. 4.5),

- other methods (Sect. 4.6).

\subsection{Cluster analysis for imprecise data}

In the last few years, in the literature, a lot of attention has focused on clustering of imprecise data. In particular, by considering on fuzzy clustering of imprecise data, we have the following contributions.

Sato and Sato (1995) proposed a fuzzy clustering procedure for fuzzy data through an additive fuzzy clustering model based on a multiple criterion. Hathaway et al. (1996) and Pedrycz et al. (1998) proposed clustering methods for fuzzy data based on, respectively, a parametric and nonparametric approach. Yang and Ko (1996) suggested fuzzy clustering methods for LR fuzzy univariate data. Yang and Liu (1999) extended the clustering techniques proposed by Yang and Ko (1996) to fuzzy vectors, i.e., conical fuzzy vectors. Takata et al. (2001) discussed the fuzzy clustering for data with uncertainties using minimum and maximum distances based on $\mathrm{L}_{1}$ metric. Auephanwiriyakul and Keller 
(2002) proposed a linguistic fuzzy clustering method for fuzzy data based on the extension principle and the decomposition theorem. Yang et al. (2004) suggested a fuzzy clustering method for mixed data, i.e., fuzzy and symbolic data, by defining a "composite" dissimilarity measure. Hung and Yang (2005) proposed a robust fuzzy clustering procedure for LR fuzzy univariate data based on exponential-type distance measure. D'Urso and Giordani (2006a) proposed a fuzzy clustering method for symmetrical fuzzy data based on a "weighted" dissimilarity measure composed of two distances: the so-called center distance and spread distance. A peculiarity of this model is the objective computation, incorporated in the clustering procedure, of weights pertaining to the center distance and spread distance of the fuzzy data. After the computation is performed, the procedure automatically evaluates the influence of the two components of the fuzzy data to compute the center and spread centroids in the fuzzy clustering. Coppi et al. (2012) proposed two clustering methods for LR fuzzy data based on, respectively, the fuzzy and the possibilistic approach. The fuzzy clustering method represents a development of the fuzzy clustering method for symmetrical fuzzy data proposed by D'Urso and Giordani (2006a). The possibilistic method is a development of the possibilistic $k$-means clustering model for crisp data proposed by Yang and $\mathrm{Wu}$ (2006).

Interesting applications of the fuzzy clustering method suggested by Coppi et al. (2012) to e-health have were pointed out by D'Urso et al. (2013a) and, following a bagged clustering approach, to tourism by D'Urso et al. (2013b, 2015a, b).

A very interesting line of research is the managing of fuzzy clustering problems in the presence of anomalous fuzzy data (Coppi et al. 2012). In this connection, different methods have been proposed in the literature.

Butkiewicz (2005) proposed the transformation of fuzzy data into crisp data and uses a classical robust fuzzy clustering method for non-fuzzy data, the so-called robust c-prototypes algorithm proposed by Frigui and Krishnapuram (1996), after artificially transforming fuzzy data into scalar data (defuzzification). In particular, the defuzzification of trapezoidal fuzzy data-represented by 4 parameters, 2 centers and 2 spreads-is obtained through the computation of the mean of these 4 parameters. All cluster prototypes produced through this optimization procedure are scalars. A re-fuzzification of the cluster prototypes is artificially produced as the mean of the original fuzzy data units, weighted with the membership degrees of each data unit to each cluster. As remarked by D'Urso and De Giovanni (2014), however, that, as a consequence of this defuzzification/re-fuzzification process, the fuzzy nature of the data is not properly taken into account in the optimization procedure.
Hung and Yang (2005) defined a robust fuzzy clustering procedure for univariate fuzzy data based on exponential distance. This is the first interesting attempt to define a robust method in which the fuzziness of the data is authentically and explicitly taken into account in the clustering process. However, this clustering method can only analyze univariate data.

Hung et al. (2010) suggested a robust clustering procedure based on a similarity measure for fuzzy data. In particular, the authors proposed a robust clustering method for fuzzy data based on the Similarity-based Clustering Method (SCM) elaborated by Yang and Wu (2004). SCM exhibits three robust clustering characteristics: (1) robust management of initialization (cluster number and initial guesses), (2) robust management of cluster volumes (ability to detect different volumes of clusters), and (3) robust management of noise and outliers. In order to achieve these objectives, the authors use a strategy for clustering LR-type fuzzy data based on a combination of three algorithms, i.e., the Correlation Comparison Algorithm (CCA), the (nonfuzzy) Similarity Clustering Algorithm (SCA), and the (non-fuzzy) Agglomerative Hierarchical Clustering (AHC) (i.e., the authors use the single linkage method). However, the clustering methods utilized in the strategy proposed by Hung et al. (2010) are not based on fuzzy theory. This means that the fuzzy data are clustered using non-fuzzy clustering methods, thus renouncing the potentiality and benefits of the fuzzy approach to cluster analysis (D'Urso 2015).

Zarandi and Razaee (2011) proposed two robust fuzzy clustering methods for fuzzy data. The first model is based on a Wasserstein-type distance and the influence weighting approach. The second model is based on a transformation that reduces the fuzzy clustering of fuzzy data to the fuzzy clustering of crisp data. Using the influence weighting approach, both methods analyze a particular type of LR fuzzy data, i.e., triangular fuzzy data. This prevents these methods from being used with other types of data belonging to the LR family, such as parabolic fuzzy data, square root fuzzy data, uniform fuzzy data, trapezoidal fuzzy data, and so on. It should also be noted that the second type of model, through a suitable adaptation of the Wasserstein-type distance utilized in the first model, reduces the fuzzy clustering problem for fuzzy data to the fuzzy clustering problem for crisp data. As such, the second method is amenable to the same criticism of the method proposed by Butkiewicz (2005). Coppi et al. (2012) introduced a clustering method for multivariate fuzzy data based on the possibilistic $k$-means clustering approach and therefore inherits the benefits of Possibilistic Theory. However, within a clustering framework, the possibilistic approach also has a number of limitations. As already noted, e.g., by 
Barni et al. (1996) and Coppi et al. (2012), an undesirable characteristic of $k$-means algorithms based on the possibilistic approach is the tendency to produce trivial solutions consisting in "coincident clusters." In other words, the algorithms may assign all prototypes to the same location. A practical work-around that is often adopted (see, e.g., Krishnapuram and Keller 1996) is to use the fuzzy $k$-means solution as starting point for possibilistic $k$-means. For the clustering of fuzzy data, Coppi et al. (2012) also recommend using the fuzzy $k$-means clustering solution as the starting point for the possibilistic $k$-means algorithm. Through a simulation study, they show that this work-around limits the risk of obtaining coincident clusters.

D'Urso and De Giovanni (2014) proposed different robust clustering methods for fuzzy data based on a suitable distance measure for fuzzy data. This measure is a metric version of the dissimilarity measure proposed by Coppi et al. (2012). In particular, using a "Partitioning Around Medoids" (PAM) approach (Kaufman and Rousseeuw 1990), D'Urso and De Giovanni first proposed a timid robustification of the fuzzy clustering of LR fuzzy data proposed by Coppi et al. (2012), i.e., the so-called Fuzzy k-Medoids Clustering method for Fuzzy data (FkMedC-F method). Successively, they proposed three robust fuzzy clustering methods, i.e., the Smoothed Fuzzy $k$-Medoids Clustering method for Fuzzy data (SFkMedC$F$ method), the Fuzzy $k$-Medoids clustering with Noise Cluster method for Fuzzy data (FkMedC-NC-F method), and the Trimmed Fuzzy $k$-Medoids Clustering method for Fuzzy data (TrFkMedC-F method). Each method neutralizes in a different manner the negative effects of outliers in the clustering process. In particular, the SFkMedC-F method achieves its robustness with respect to outliers by taking into account a "robust" distance measure; the FkMedC-NC-F method achieves its robustness with respect to outliers by introducing a noise cluster represented by a noise prototype and the TrFkMedC-F method achieves its robustness with respect to outliers by trimming away a certain fraction of the data units.

For a survey on fuzzy clustering methods for fuzzy data, see D'Urso (2007).

Notice that, in the literature, there are various clustering methods proposed for classifying imprecise data that are modeled as interval-valued data. In this case, the interval algebra is used for formalizing these kinds of data. In a fuzzy framework, the interval-valued data can be seen as fuzzy data with uniform membership function. In this connection, interesting contributions on (nonfuzzy) clustering of interval-valued data have been proposed, e.g., by de Sousa and de Carvalho (2004), Guru et al. (2004), de Carvalho et al. (2006a, b), de Carvalho and Lechevallier (2009a, b), Hardy and Kasaro (2009), de Carvalho and de Souza (2010), and Kao et al. (2014).

Attention has also been focused on fuzzy clustering of interval-valued data (see, e.g., El-Sonbaty and Ismail 1998; D'Urso and Giordani 2006a, b; de Carvalho 2007; de Carvalho and Tenorio 2010; D'Urso et al. 2015a, b). In this regards, an interesting line of research is that of the managing of fuzzy clustering in the presence of outlier interval-valued data, i.e., robust fuzzy clustering (D’Urso and Giordani 2006a; D’Urso et al. 2015). For interval-valued data, according to our knowledge, only four robust fuzzy clustering methods have been proposed in the literature, i.e., the noise approach-based fuzzy clustering proposed by D'Urso and Giordani (2006b), the trimmed approachbased fuzzy clustering proposed by D'Urso et al. (2015), the exponential distance-based fuzzy clustering proposed by D'Urso et al. (2017), and the fuzzy c-ordered-medoids clustering for interval-valued data proposed by D'Urso and Leski (2016) that is a Partitioning Around Medoids (PAM) fuzzy clustering method based on Huber's M-estimators and Yager's OWA (Ordered Weighted Averaging) operators.

\subsubsection{A representative example}

As an example, we here illustrate schematically the fuzzy clustering for LR fuzzy data proposed by Coppi et al. (2012), which is characterized by the following steps:

\section{1. input data and distance measure; \\ 2. clustering procedure.}

4.1.1.1 Input data and distance measure The input data are represented by Eqs. (1)-(2). However, prior to computing the distance measure and to applying the clustering model, it may be advisable to preprocess the data in order to eliminate unwanted differences among the variables. The LR fuzzy data can be pre-processed as follows. Taking into account that each LR fuzzy number is characterized by two "centers" and two spreads, it is possible to standardize simultaneously the "centers" by considering the mean and the standard deviation of both "centers." Thus, each left and right spread is divided by the standard deviation of the corresponding "centers." By means of this pre-processing step, it is possible to remove artificial range differences while keeping the information about the relative sizes of the spreads (Coppi et al. 2006a).

In order to measure the dissimilarity between each pair of objects, i.e., the observed data and the estimated ones, Coppi et al. (2012) proposed a distance measure for LR fuzzy data, which is an extension of the distance suggested by D'Urso and Giordani (2006a), i.e., 


$$
\begin{aligned}
{ }_{C D G} d\left(\tilde{\mathbf{x}}_{i}, \tilde{\mathbf{x}}_{i^{\prime}}\right)= & {\left[w_{C}^{2}\left[d^{2}\left(\mathbf{c}_{1 i}, \mathbf{c}_{1 i^{\prime}}\right)+d^{2}\left(\mathbf{c}_{2 i}, \mathbf{c}_{2 i^{\prime}}\right)\right]\right.} \\
& \left.+w_{S}^{2}\left[d^{2}\left(\mathbf{l}_{i}, \mathbf{l}_{i^{\prime}}\right)+d^{2}\left(\mathbf{r}_{i}, \mathbf{r}_{i^{\prime}}\right)\right]\right]^{\frac{1}{2}},
\end{aligned}
$$

where $d\left(\mathbf{c}_{1 i}, \mathbf{c}_{1 i^{\prime}}\right)=\left\|\mathbf{c}_{1 i}-\mathbf{c}_{1 i^{\prime}}\right\|$ is the Euclidean distance between the left centers $\mathbf{c}_{1 i}$ and $\mathbf{c}_{1 i^{\prime}} ; d\left(\mathbf{c}_{2 i}, \mathbf{c}_{2 i^{\prime}}\right)=\left\|\mathbf{c}_{2 i}-\mathbf{c}_{2 i^{\prime}}\right\|$ is the Euclidean distance between the right centers $\mathbf{c}_{2 i}$ and $\mathbf{c}_{2 i^{\prime}} ; d\left(\mathbf{l}_{i}, \mathbf{l}_{i^{\prime}}\right)=\left\|\mathbf{l}_{i}-\mathbf{l}_{i^{\prime}}\right\|$ is the Euclidean distance between the left spreads $\mathbf{l}_{i}$ and $\mathbf{l}_{i^{\prime}} ; d\left(\mathbf{r}_{i}, \mathbf{r}_{i^{\prime}}\right)=\left\|\mathbf{r}_{i}-\mathbf{r}_{i^{\prime}}\right\|$ is the Euclidean distance between the right spreads $\mathbf{r}_{i}$ and $\mathbf{r}_{i}$; $\mathbf{c}_{1 i} \equiv\left(c_{1 i 1}, \ldots, c_{1 i j} \ldots, c_{1 i p}\right)^{\prime}, \quad \mathbf{c}_{1 i^{\prime}} \equiv\left(c_{1 i^{\prime} 1}, \ldots, c_{1 i^{\prime} j} \ldots, c_{1 i^{\prime} p}\right)^{\prime}$, $\mathbf{c}_{2 i} \equiv\left(c_{2 i 1}, \ldots, c_{2 i j} \ldots, c_{2 i p}\right)^{\prime}, \quad \mathbf{c}_{2 i^{\prime}} \equiv\left(c_{2 i^{\prime} 1}, \ldots, c_{2 i^{\prime} j} \ldots, c_{2 i^{\prime} p}\right)^{\prime}$, $\mathbf{l}_{i} \equiv\left(l_{i 1}, \ldots, l_{i j} \ldots, l_{i p}\right)^{\prime}, \quad \mathbf{l}_{i^{\prime}} \equiv\left(l_{i^{\prime}}, \ldots, l_{i^{\prime} j} \ldots, l_{i^{\prime} p}\right)^{\prime}$, $\mathbf{r}_{i} \equiv\left(r_{i 1}, \ldots, r_{i j} \ldots, r_{i p}\right)^{\prime}, \mathbf{r}_{i^{\prime}} \equiv\left(r_{i^{\prime} 1}, \ldots, r_{i^{\prime} j} \ldots, r_{i^{\prime} p}\right)^{\prime} ; w_{C}, w_{S} \geq 0$ are suitable weights-computed objectively within the clustering procedure-for the center component and the spread component of ${ }_{C D G} d\left(\tilde{\mathbf{x}}_{i}, \tilde{\mathbf{x}}_{i^{\prime}}\right)$, where $\tilde{\mathbf{x}}_{i}$ and $\tilde{\mathbf{x}}_{i^{\prime}}$ denote the fuzzy data vectors, respectively, for the $i$-th and $i$ '-th objects, i.e., $\tilde{\mathbf{x}}_{i} \equiv\left\{\tilde{x}_{i j}=\left(c_{1 i j}, c_{2 i j}, l_{i j}, r_{i j}\right)_{L R}: j=1, \ldots, p\right\}$ and $\tilde{\mathbf{x}}_{i^{\prime}} \equiv\left\{\tilde{x}_{i}{ }_{j}=\left(c_{1 i},{ }_{j}, c_{2, i},{ }_{j}, l_{i},{ }_{j}, r_{i},{ }_{j}\right)_{L R}: j=1, \ldots, p\right\}$.

D'Urso, De Giovanni and Massari (2014) proved that the distance shown in Eq. (6) satisfy the metric properties.

\subsubsection{Clustering procedure Coppi et al. (2012) pro-} posed the following fuzzy clustering method for LR fuzzy multivariate data:
By solving the constrained quadratic minimization problem shown in Eq. (7) via Lagrangian multiplier method with respect to $u_{i g}$ and by setting the first derivatives of $J_{\mathrm{F} k \mathrm{M}-\mathrm{LR}}$ with respect to $\mathbf{h}_{g}^{C_{1}}, \mathbf{h}_{g}^{C_{2}}, \mathbf{h}_{g}^{L}, \mathbf{h}_{g}^{R}$ and $\mathbf{w}$ equal to zero, Coppi et al. derived the iterative solution (see, Coppi et al. 2012).

Notice that, in the clustering method shown in Eq. (7), before computing the membership degrees and the centroids iteratively, the number of clusters $k$ has to be fixed. Cluster validity criteria that will can be used are the XieBeni criterion (Xie and Beni 1991) and the silhouette criterion (Campello and Hruschka 2006). Furthermore, in order to display the obtained optimal partition, different graphical procedures can be considered, e.g., silhouette plot (Campello and Hruschka 2006), ternary plot, Modified Sammon Mapping; for other visualization procedures, see D’Urso (2015).

We observe that, by considering the same metric shown in Eq. (6), Coppi et al. (2012) also suggested a possibilistic version of the method shown in Eq. (7). Method shown in Eq. (7) represents an extension of the D'Urso-Giordani model (2006a) proposed for a particular case of LR fuzzy data.

$$
\begin{aligned}
& \min _{u_{i g}, \mathbf{h}_{g}, \mathbf{w}}: J_{\mathrm{F} k \mathrm{M}-L R} \equiv \sum_{i=1}^{n} \sum_{g=1}^{k} u_{i g}^{m} C D G \\
& d^{2}\left(\tilde{\mathbf{x}}_{i}, \tilde{\mathbf{h}}_{g}\right)=\sum_{i=1}^{n} \sum_{g=1}^{k} u_{i g}^{m}\left[w_{C}^{2}\left[d^{2}\left(\mathbf{c}_{1 i}, \mathbf{h}_{g}^{C_{1}}\right)+d^{2}\left(\mathbf{c}_{2 i}, \mathbf{h}_{g}^{C_{2}}\right)\right]+w_{S}^{2}\left[d^{2}\left(\mathbf{l}_{i}, \mathbf{h}_{g}^{L}\right)+d^{2}\left(\mathbf{r}_{i}, \mathbf{h}_{g}^{R}\right)\right]\right], \\
& \text { s.t. } u_{i g} \in[0,1], \sum_{g=1} u_{i g}=1, \\
& \mathbf{w} \equiv\left(w_{C} w_{S}\right)^{\prime} \geq \mathbf{0}_{2}, w_{C} \geq w_{S}, w_{C}+w_{S}=1,
\end{aligned}
$$

where $m>1$ is a weighting exponent that controls the fuzziness of the obtained partition (see D'Urso et al. 2014);

$u_{i g}$ indicates the membership degree of the $i$-th object in the $g$-th cluster;

${ }_{C D G} d^{2}\left(\tilde{\mathbf{x}}_{i}, \tilde{\mathbf{h}}_{g}\right)$ represents the suggested dissimilarity measure between the $i$-th object and the prototype of the $g$-th cluster, analogously for its components $d^{2}\left(\mathbf{c}_{1 i}, \mathbf{h}_{g}^{C_{1}}\right), d^{2}\left(\mathbf{c}_{2 i}, \mathbf{h}_{g}^{C_{2}}\right), d^{2}\left(\mathbf{l}_{i}, \mathbf{h}_{g}^{L}\right), d^{2}\left(\mathbf{r}_{i}, \mathbf{h}_{g}^{R}\right)$, where the fuzzy vector $\tilde{\mathbf{h}}_{g} \equiv\left\{\tilde{h}_{g j}=\left(h_{g}^{C_{1}}, h_{g}^{C_{2}}, h_{g}^{L}, h_{g}^{R}\right)_{L R}: j=1, \ldots, p\right\}$ represents the fuzzy prototype of the $g$-th cluster; $\mathbf{h}_{g}^{C_{1}} \equiv\left(h_{g 1}^{C_{1}}, \ldots, h_{g j}^{C_{1}}, \ldots h_{g p}^{C_{1}}\right)^{\prime}, \quad \mathbf{h}_{g}^{C_{2}} \equiv\left(h_{g 1}^{C_{2}}, \ldots, h_{g j}^{C_{2}}, \ldots h_{g p}^{C_{2}}\right)^{\prime}$, $\mathbf{h}_{g}^{L} \equiv\left(h_{g 1}^{L}, \ldots, h_{g j}^{L}, \ldots h_{g p}^{L}\right)^{\prime}$, and $\mathbf{h}_{g}^{R} \equiv\left(h_{g 1}^{R}, \ldots, h_{g j}^{R}, \ldots h_{g p}^{R}\right)^{\prime}$ are $p$-vectors, whose $j$-th element refers to the $j$-th variable, that denote, respectively, the (left and right) centers and the (left and right) spreads of the $g$-th fuzzy prototype.

\subsection{Self-organizing maps (SOMs) for imprecise data}

In the literature, there are various examples of Self-Organizing Maps (SOMs) for imprecise data modeled as interval and symbolic data.

Bock $(1999,2008)$ proposed to visualize symbolic data (i.e., interval-valued data) by using SOMs. Chen et al. (2010) suggested a batch version of the SOMs for symbolic data. D'Urso and De Giovanni (2011) proposed Midpoint Radius-Based Self-Organizing Maps (MR-SOMs) for interval-valued data showing a suggestive telecommunications application. Hajjar and Hamdan proposed a selforganizing map for individuals-variables interval data using $\mathrm{L}_{2}$ distance (Hajjar and Hamdan 2011a; Hamdan and Hajjar 2011), Hausdorff distance (Hajjar and Hamdan 2011b), City block distance (Hajjar and Hamdan 2012), an extension of $\mathrm{L}_{2}$ distance for interval data (Hamdan and Hajjar 2012), and Mahalanobis distance (Hajjar and Hamdan 2013). Yang 
et al. (2012) suggested SOMs for symbolic data. Cabanes et al. (2013) proposed a clustering algorithm for interval data, based on the learning of a self-organizing map. The algorithm suggested by Cabanes et al. (2013) is a two-level clustering method for interval data. The key idea of the twolevel clustering approach based on SOM is to combine the dimension reduction and the fast learning capabilities of SOM in the first level to construct a new reduced space, then to apply a clustering method in this new space to produce a final set of clusters in the second level. El Golli et al. (2004) defined an adaptation of the self-organizing map to interval-valued dissimilarity data by implementing the SOM algorithm on interval-valued dissimilarity measures rather than on individuals-variables interval data.

According to our knowledge, the only work on SOMs for data imprecisely observed in which the imprecise data are modeled in a fuzzy manner D'Urso et al. (2014). D'Urso et al. (2014) proposed an extension of the SOMs for fuzzy data in which the learning algorithm is based on two distances for fuzzy data. The proposed SOMs allow clustering and vector quantization of fuzzy data.

\subsubsection{A representative example}

As an example, we illustrate the SOM for LR fuzzy number-valued data (SOMF-LR) proposed by D'Urso et al. (2014) based on the distance shown in Eq. (3) and a multivariate extension of the Yang-Ko distance (1996). This can be divided in following steps:

\section{1. input data and distance measure;}

2. model, learning rule, and quality of learning.

4.2.1.1 Input data and distance measure The input data are represented by Eqs. (1)-(2). For a possible data pre-processing, see sub-Sect. 4.1.1.

The distance measured utilizing the self-organizing maps is shown in Eq. (6).

\subsubsection{Model, learning rule, quality of learning, and plot-} ting procedure The SOM is a network (topology, lattice) of $q$ functional units or neurons arranged in a one-dimensional or multidimensional configuration. Each neuron $p$, $1 \leq q \leq \tilde{q}$ has a (scalar or vectorial) location (coordinate) $r_{q}$ dependent on the configuration (one-dimensional or multidimensional), and an initial $p$-dimensional weight $\varphi_{q}=$ $\left(\varphi_{q 1}, \ldots \varphi_{q s}, \ldots, \varphi_{q p}\right)$. Then, there is a set of $n p$-dimensional input vectors $\xi_{i}=\left(\xi_{i l}, \ldots, \xi_{i s}, \ldots, \xi_{i p}\right)$. At ordering step $t$, an input vector $\xi_{i}(t)$ is compared in any metric with the weight vectors and the winner neuron $\tilde{c}$ (response or best matching unit, $b m u)$ whose weight vector $\varphi_{\tilde{c}}$ is closest to $\xi_{i}(t)$ is selected. The learning rule of the weights is the following (Kohonen 1995):

$\varphi_{q}(t+1)=a(t) \xi_{i}(t)+(1-a(t)) \varphi_{q}(t)$

$\varphi_{q}(t+1)=\varphi_{q}(t)$

if $q \in N_{\tilde{c}}$ otherwise

where $\alpha(t)$ is the learning rate and $N_{\tilde{c}}=N_{\tilde{c}}(t)$ is the topological neighborhood of $\varphi_{\tilde{c}}$.

The neighborhood is often given in terms of a neighborhood function. In this case, the learning rule of the weights is the following:

$\varphi_{q}(t+1)=a(t) h_{q, i}(t) \xi_{i}(t)+\left(1-a(t) h_{q, i}(t)\right) \varphi_{q}(t)$

where $h_{q, i}(t)$ is the neighborhood function measuring the distance between the locations of neuron $q$ and closest (winner) neuron $\tilde{c}$ to the input vector $\xi_{i}(t)$. A frequently used form for neighborhood function is $h_{q, i}(t)=\exp \left(-\frac{\left\|\tilde{\mathbf{r}}_{q}-\tilde{\mathbf{r}}_{\tilde{c}}\right\|^{2}}{2 \sigma(t)^{2}}\right)$, where $\tilde{\mathbf{r}}_{q}$ and $\tilde{\mathbf{r}}_{\tilde{c}}$ identify the locations (coordinates) of neurons $q$ and $\tilde{c}$ in the configuration (topology) and $\sigma(t)$ is the (decreasing) width of the neighborhood.

According to Kohonen (1995), the randomly chosen initial values for the $\varphi_{q}$ gradually change to new values in a learning process specified by Eq. (5) or Eq. (6) such that, as $t \rightarrow \infty$, the weight vectors of the neurons $\boldsymbol{\varphi}_{1}, \ldots, \boldsymbol{\varphi}_{q}, \ldots, \boldsymbol{\varphi}_{\tilde{q}}$ become ordered (neurons with nearest location exhibit smaller distance with respect to weights), and that the probability density function of the weight vectors finally approximates some monotonic function of the probability density function $f(\boldsymbol{\xi})$ of the $p$-dimensional continuous random variable $\xi$.

Considering the SOM algorithm shown in Eq. (9), in order to use the SOM for clustering and vector quantization of LR fuzzy data, the winner is selected on the basis of the distance shown in Eq. (6). According to Eq. (6), the distance considered between the weight vector $\boldsymbol{\varphi}_{q}(t)$ and the generic input vector $i$ sorted for updating the SOM at ordering step $t, \boldsymbol{\xi}_{i}(t)=\tilde{\mathbf{x}}_{i}$ is

$$
\begin{aligned}
& C D G d\left(\boldsymbol{\varphi}_{q}(t), \boldsymbol{\xi}_{i}(t)\right) \approx \\
& \left(w_{C}^{2}\left(\left\|\boldsymbol{\varphi}_{1 q}(t)-\mathbf{c}_{1 i}(t)\right\|^{2}+\left\|\boldsymbol{\varphi}_{2 q}(t)-\mathbf{c}_{2 i}(t)\right\|^{2}\right)+w_{S}^{2}\left(\left\|\boldsymbol{\varphi}_{l q}(t)-\mathbf{l}_{i}(t)\right\|^{2}+\left\|\boldsymbol{\varphi}_{r q}(t)-\mathbf{r}_{i}(t)\right\|^{2}\right)\right)^{1 / 2}= \\
& \left((1-v(t))^{2}\left(\left\|\boldsymbol{\varphi}_{1 q}(t)-\mathbf{c}_{1 i}(t)\right\|^{2}+\left\|\boldsymbol{\varphi}_{2 q}(t)-\mathbf{c}_{2 i}(t)\right\|^{2}\right)+v(t)^{2}\left(\left\|\boldsymbol{\varphi}_{l q}(t)-\mathbf{l}_{i}(t)\right\|^{2}+\left\|\boldsymbol{\varphi}_{r q}(t)-\mathbf{r}_{i}(t)\right\|^{2}\right)\right)^{1 / 2}
\end{aligned}
$$


where $\boldsymbol{\varphi}_{q}(t)$ is the $p$-dimensional vector of left and right centers and left and right spreads for the weight vector of neuron $q$ in which $\boldsymbol{\varphi}_{1 q}(t), \boldsymbol{\varphi}_{2 q}(t), \boldsymbol{\varphi}_{l q}(t)$, and $\boldsymbol{\varphi}_{r q}(t)$ are the $p$-dimensional vectors of left centers, right centers, left spreads, and right spreads, respectively.

The quality of learning in the SOM is measured through the average expected quantization error and the average expected distortion measure that are, respectively,

$$
\int_{\mathfrak{R}^{p}} C D G d\left(\boldsymbol{\varphi}_{\tilde{c}}, \boldsymbol{\xi}\right) f(\boldsymbol{\xi})
$$

$$
\int_{\mathfrak{R}^{p}} \sum_{q=1}^{\tilde{q}} h_{q, \boldsymbol{\xi}}(t)_{C D G} d^{2}\left(\boldsymbol{\varphi}_{q}, \boldsymbol{\xi}\right) f(\boldsymbol{\xi})
$$

where $\xi \in \Re^{p}$ is the input vector, $\varphi_{\tilde{c}}$ is the weight vector closest to the input vector $\xi$ according to ${ }_{C D G} d$, and $h_{q, \xi}(t)$ is the degree of neighborhood between the locations of neuron $q$ and winner neuron $\tilde{c}$.

The SOM algorithm might be derivable from the optimization (minimization) of the average expected distortion measure by computing the gradient of the measure shown in Eq. (12) (Kohonen 1995). The average quantization error and the average distortion measure are the sample counterparts of Eqs. (8) and (9) and are defined as follows:

$$
\begin{aligned}
& n^{-1} \sum_{i=1}^{n}{ }_{C D G} d\left(\boldsymbol{\varphi}_{\tilde{c}}, \boldsymbol{\xi}_{i}\right) \\
& \sum_{i=1}^{I} \sum_{q=1}^{\tilde{q}} h_{q, i}(t)_{C D G} d^{2}\left(\boldsymbol{\varphi}_{q}, \boldsymbol{\xi}_{i}\right) .
\end{aligned}
$$

In order to improve the quality of learning, the value of $\nu$ is determined so as to minimize the average distortion measure shown in Eq. (14). By computing the derivative of the measure shown in Eq. (14) with respect to $v(t)$, the optimum value of $v(t)$ becomes $\boldsymbol{\varphi}_{1 q}(t+1)=a(t) h_{q, i}(t) \mathbf{c}_{1 i}(t)+\left(1-a(t) h_{q, i}(t)\right) \boldsymbol{\varphi}_{1 q}(t)$

$\boldsymbol{\varphi}_{2 q}(t+1)=a(t) h_{q, i}(t) \mathbf{c}_{2 i}(t)+\left(1-a(t) h_{q, i}(t)\right) \boldsymbol{\varphi}_{2 q}(t)$

$\boldsymbol{\varphi}_{l q}(t+1)=a(t) h_{q, i}(t) \mathbf{l}_{i}(t)+\left(1-a(t) h_{q, i}(t)\right) \boldsymbol{\varphi}_{l q}(t)$

$\boldsymbol{\varphi}_{r q}(t+1)=a(t) h_{q, i}(t) \mathbf{r}_{i}(t)+\left(1-a(t) h_{q, i}(t)\right) \boldsymbol{\varphi}_{r q}(t)$

where $\tilde{c}$ is the neuron closest to $\xi_{i}(t)$.

For a formalization of the SOMF-LR based on the multivariate extension of the Yang-Ko distance, see D'Urso et al. (2014).

\subsection{Regression analysis for imprecise data}

Regression analysis constitutes one of the most popular methodological areas for the use of fuzzy sets theory to manage imprecision affecting empirical data. It is certainly the most prolific area in terms of scientific productivity as there are several papers published on regression analysis with fuzzy variables (i.e., fuzzy-dependent variables and/or fuzzy explanatory variables).

In particular, in the literature, different approaches to regression analysis for fuzzy data have been developed, starting from the pioneering works by Tanaka et al. (1982), Celminš (1987), Diamond (1988), based respectively on possibilistic principles (Tanaka et al. 1993) and least squares principles. In this connection, two main approaches are available:

- The possibilistic approach In this framework, given a regression model involving fuzzy regression coefficients, these are estimated by minimizing the fuzziness of the estimated response variable, conditionally on obtaining fuzzy response values which contain (to a certain possibility degree) the observed fuzzy responses. Several papers have been published in this area (see, for instance, Tanaka et al. 1982; Tanaka and Watada 1988; and, in a comparative perspective; Kim et al. 1996; Dia-

$v(t)=\min \left\{\frac{\sum_{i=1}^{n} \sum_{q=1}^{\tilde{q}} h_{q, i}(t)\left(\left\|\boldsymbol{\varphi}_{1 q}(t)-\mathbf{c}_{1 i}(t)\right\|^{2}+\left\|\boldsymbol{\varphi}_{2 q}(t)-\mathbf{c}_{2 i}(t)\right\|^{2}\right)}{\sum_{i=1}^{n} \sum_{q=1}^{\tilde{q}} h_{q, i}(t)\left(\left\|\boldsymbol{\varphi}_{1 q}(t)-\mathbf{c}_{1 i}(t)\right\|^{2}+\left\|\boldsymbol{\varphi}_{2 q}(t)-\mathbf{c}_{2 i}(t)\right\|^{2}+\left\|\boldsymbol{\varphi}_{l q}(t)-\mathbf{l}_{i}(t)\right\|^{2}+\left\|\boldsymbol{\varphi}_{r q}(t)-\mathbf{r}_{i}(t)\right\|^{2}\right)}, 0.5\right\}$.

Considering distance shown in Eq. (6), the updating rules for the weight vector $\varphi_{q}(t)$ when the generic input vector $i$ is sorted for updating the SOMs at ordering step $t$ $\boldsymbol{\xi}_{i}(t)=\tilde{\mathbf{x}}_{i}$ are mond and Tanaka 1998; Chang and Ayyub 2001).

- The least squares approach This approach is based on suitable extensions of the well-known least squares criterion to the fuzzy setting. It aims at finding a linear 
model which "best approximates" the observed data in a given metric space. The least squares criterion is then utilized with respect to the chosen metric. This approach has been followed by many researchers in the last two decades (for instance, Celminš 1987; Diamond 1988; Chang and Lee 1996; Ma et al. 1997; D’Urso and Gastaldi 2000, 2002; Coppi and D'Urso 2003a, b; D'Urso 2003; Wu 2003; Coppi et al. 2006c; D'Urso and Santoro 2006a, b; D'Urso et al. 2011; Takemura, 2011; D'Urso and Massari 2013). However, the proposals made in the papers devoted to this line of thought are very heterogeneous. The main features distinguishing the different suggestions are represented by the definition of the linear regression model, on one side, and, on the other side, by the specific metric space introduced for applying the least squares criterion (Coppi et al. 2006a, b, c, d).

Since several contributions belonging to two previous approaches have been proposed in the literature, we refer to interested readers the journals devoted to this topic, such as Fuzzy Sets and Systems, IEEE Transactions on Fuzzy Systems, Information Sciences, Applied Soft Computing, Soft Computing, and Computational Statistics and Data Analysis.

\subsubsection{A representative example}

As an example, in the following, we illustrate schematically the so-called weighted least squares approach-based fuzzy linear regression analysis for studying the relationship between a LR fuzzy response variable and a set of LR fuzzy explanatory variables, proposed by D'Urso and Massari (2013). This represents a generalization of the fuzzy regression analysis proposed by Coppi et al. (2006c) and Coppi and D'Urso (2003a, b). D'Urso and Massari (2013) also proposed a robust version that is an extension of the robust model proposed by D'Urso et al. (2011). All these least squares-based regression models are inspired by the following simple idea (D'Urso and Gastaldi 2000, 2002; D'Urso 2003): the general fuzzy regression model is based on different sub-models. The first one interpolates the centers of the fuzzy data, the other sub-models are built over the first one and yield the spreads. This formulation allows the model to consider possible relations between the size of the spreads and the magnitude of the estimated centers, as it is often necessary in real case studies.

Schematically, the steps of the fuzzy regression analysis suggested by D'Urso and Massari (2013) are the following:

\footnotetext{
1. input data and distance measure,

2. model.
}

4.3.1.1 Input data and distance measure LR fuzzy data are considered in the regression analysis, i.e., LR fuzzy response variable $\tilde{Y}=\left(c_{1}, c_{2}, l, r\right)_{L R}$ and a set of $p$ LR fuzzy explanatory variables $\tilde{X}_{s}=\left(c_{1 s}, c_{2 s}, l_{s}, r_{s}\right)_{L R}, s=1, \ldots p$.

For the data pre-processing, see sub-sect. 4.1.1.

The metric considered in the least squares estimation process is a multivariate extension of the Yang-Ko distance (1996).

4.3.1.2 Model The linear regression model for a fuzzy response variable $\tilde{Y}=\left(c_{1}, c_{2}, l_{i}, r_{i}\right)_{L R}$ and a set of $p$ LR fuzzy explanatory variables $\tilde{X}_{s}=\left(c_{1 s}, c_{2 s}, l_{i s}, r_{i s}\right)_{L R}, s=1$, ... $p$ is

$$
\begin{array}{cc}
\mathbf{c}_{1}=\mathbf{c}_{1}^{*}+\boldsymbol{\varepsilon}_{\mathbf{c}_{1}} & \mathbf{c}_{1}^{*}=\mathbf{C}_{1} \boldsymbol{\alpha}_{1}+\mathbf{C}_{2} \boldsymbol{\alpha}_{2}+\mathbf{L} \boldsymbol{\alpha}_{l}+\mathbf{R} \boldsymbol{\alpha}_{r} \\
\mathbf{c}_{2}=\mathbf{c}_{2}^{*}+\boldsymbol{\varepsilon}_{\mathbf{c}_{2}} & \mathbf{c}_{2}^{*}=\mathbf{C}_{1} \boldsymbol{\beta}_{1}+\mathbf{C}_{2} \boldsymbol{\beta}_{2}+\mathbf{L} \boldsymbol{\beta}_{l}+\mathbf{R} \boldsymbol{\beta}_{r} \\
\mathbf{l}=\mathbf{l}^{*}+\boldsymbol{\varepsilon}_{l} & \mathbf{l}^{*}=\mathbf{1} \gamma_{0}+\mathbf{c}_{1}^{*} \gamma_{1}+\mathbf{c}_{2}^{*} \gamma_{2}=\mathbf{C}^{*} \boldsymbol{\gamma} \\
\mathbf{r}=\mathbf{r}^{*}+\boldsymbol{\varepsilon}_{r} & \mathbf{r}^{*}=\mathbf{1} \delta_{0}+\mathbf{c}_{1}^{*} \delta_{1}+\mathbf{c}_{2}^{*} \delta_{2}=\mathbf{C}^{*} \boldsymbol{\delta}
\end{array}
$$

where

$\mathbf{c}_{1}, \mathbf{c}_{2}$ are the $n$-vectors of the left and right centers of the fuzzy response variable;

$\mathbf{I}, \mathbf{r}$ are the $n$-vectors of the left and right spreads of the fuzzy response variable;

$\mathbf{C}_{1}, \mathbf{C}_{2}$ are the $(n \times(p+1))$-matrices of the left and right centers of the fuzzy input variables containing a vector of ones;

$\mathbf{L}, \mathbf{R}$ are the $(n \times(p+1))$-matrices of the left and right spreads of the fuzzy input variables containing a vector of ones;

$\boldsymbol{\alpha}_{1}, \boldsymbol{\alpha}_{2}, \boldsymbol{\alpha}_{l}$, and $\boldsymbol{\alpha}_{r}$ are the $(p+1)$-vectors of coefficients of the models on the left centers $\mathbf{c}_{1}$;

$\boldsymbol{\beta}_{1}, \boldsymbol{\beta}_{2}, \boldsymbol{\beta}_{l}$ and $\boldsymbol{\beta}_{r}$ are the $(p+1)$-vectors of coefficients of the models on the right centers $\mathbf{c}_{2}$;

$\gamma_{0}, \gamma_{1}$, and $\gamma_{2}$ are the coefficients of the models on the left spreads, $\mathbf{l}$;

$\delta_{0}, \delta_{1}$, and $\delta_{2}$ are their counterparts for the model on the right spreads, $\mathbf{r}$;

$\boldsymbol{\varepsilon}_{\mathbf{c}_{1}}, \boldsymbol{\varepsilon}_{\mathbf{c}_{2}}$ are the $n$-vectors of the error terms of the models on the left and right centers;

$\boldsymbol{\varepsilon}_{l}, \boldsymbol{\varepsilon}_{r}$ are the $n$-vectors of the residual terms of the models on the left and right spreads;

1 is the $n$-vector of ones;

the theoretical values of the centers and spreads are marked with an asterisk symbol (*);

$\mathbf{C}^{*}$ is the $(n \times 3)$ matrix whose columns are the vector of ones and the vectors of the theoretical values of the left and right centers of the response variable;

$\gamma$ and $\delta$ are the $(3 \times 1)$ vectors of the coefficients of the model on the left and right spreads.

Notice that in order to neutralize possible problems connected to the negativity of estimated spreads suitable solutions can be adopted, e.g., considering the logarithmic 
transformation of the spreads (see D'Urso 2003; D'Urso and Massari 2013).

By adopting the multivariate version of the Yang-Ko metric, the objective function to be minimized is the weighted squared Euclidean distance between the observed fuzzy variables and their estimates is

$$
\begin{aligned}
\tilde{\Delta}_{\mathbf{W}}^{2}= & \left\|\mathbf{c}_{1}-\mathbf{c}_{1}^{*}\right\|_{\mathbf{W}}^{2}+\left\|\mathbf{c}_{2}-\mathbf{c}_{2}^{*}\right\|_{\mathbf{W}}^{2} \\
& +\left\|\left(\mathbf{c}_{1}-\lambda \mathbf{l}\right)-\left(\mathbf{c}_{1}^{*}-\lambda \mathbf{l}^{*}\right)\right\|_{\mathbf{W}}^{2} \\
& +\left\|\left(\mathbf{c}_{2}+\rho \mathbf{r}\right)-\left(\mathbf{c}_{2}^{*}+\rho \mathbf{r}^{*}\right)\right\|_{\mathbf{W}}^{2}
\end{aligned}
$$

where the parameters $\lambda=\int_{0}^{1} L^{-1}(\omega) d \omega, \rho=\int_{0}^{1} R^{-1}(\omega) d \omega$ have the twofold role of taking into account the variability of the membership function and decreasing the emphasis on the spreads (Coppi et al. 2006c; D'Urso and Massari 2013), and $\mathbf{W}$ is a diagonal matrix, whose elements are the weights attached to each observation.

By minimizing the objective function shown in Eq. (18), D'Urso and Massari (2013) obtained the iterative solution of the model shown in Eq. (17).

Suitable indices for measuring the goodness of fit have been suggested by D'Urso and Massari (2013). See also Coppi et al. (2006c) and D'Urso and Santoro (2006a).

\subsection{Principal component analysis for imprecise data}

Several contributions have been proposed for Principal Component Analysis (PCA) in a fuzzy domain, e.g., Yabuuch and Watada (1997), Yang and Wang (2000), Sarbu and Pop (2005), Honda and Ichihashi (2006), Yang et al. (2008), Pop et al. (2009), Heo et al. (2009), Honda et al. (2010), Rezaee and Moini (2013), Xian et al. (2013), and Baklouti et al. (2016).

With respect to the specific area of PCA for imprecise data, we draw attention to the following contributions: Denœux and Masson (2004), D'Urso and Giordani (2004, 2005), Giordani and Kiers (2004a, b, 2006), Coppi et al. (2006a, b, c, d), Giordani (2006, 2010), Douzal-Chouakria et al. (2011), Le-Rademacher, Billard (2012), Liu et al. (2014, 2015), and Calcagnì et al. (2016). In particular, Denœux and Masson (2004) suggested a PCA of fuzzy data using autoassociative neural networks. D'Urso and Giordani (2004) proposed a least squares-based PCA for imprecise data, i.e., interval-valued data. The same authors proposed a PCA for fuzzy data based on a possibilistic approach (D'Urso and Giordani 2005). Giordani and Kiers (2004a) suggested a PCA for LR fuzzy data based on the least squares approach. The same authors (Giordani and Kiers 2006) compared some PCA methods for fuzzy data. Coppi et al. (2006a) proposed a new PCA for fuzzy data. In particular, in Coppi et al. (2006a), the problem of detecting the underlying structure of LR fuzzy data is dealt with. The basic inspiration is the PCA decomposition. In particular, they look at this decomposition from a least squares viewpoint. This perspective implies finding component matrices such that the overall distance between observed and modeled data is minimized. This task must be achieved while trying to reduce as much as possible the number of necessary dimensions for approximating the observed data. Liu et al. (2014, 2015b) suggested a group decision-making method based on the interval-valued intuitionistic fuzzy PCA. Calcagnì et al. (2016) proposed a dimension reduction technique for two-mode non-convex fuzzy data.

See Denœux and Masson (2007) for a brief and interesting survey on dimensionality reduction and visualization of interval and fuzzy data, i.e., PCA and multidimensional scaling (see Sect. 4.5).

\subsubsection{A representative example}

By way of example, we illustrate schematically the Principal Component Analysis for Fuzzy LR data (PCAF-LR) proposed by Coppi et al. 2006a, considering the following steps:

1. input data and distance measure,

2. model.

4.4.1.1 Input data and distance measure The input data are represented by Eqs. (1)-(2). Also in the PCAF-LR prior to fitting the model, it may be advisable to preprocess the data. To this purpose, we suggest following the same indications shown in sub-sect. 4.1.1.

In order to compare the observed data and the estimated ones, Coppi et al. (2006a) considered the following multivariate extension of the Yang-Ko distance for LR fuzzy data (Yang and Ko 1996):

$$
\begin{aligned}
& { }_{Y K} d_{L R}^{2}=\left\|\mathbf{C}_{1}-\mathbf{C}_{1}^{*}\right\|^{2}+\left\|\mathbf{C}_{2}-\mathbf{C}_{2}^{*}\right\|^{2}+ \\
& +\sum_{s=1}^{v}\left\|\left[\left(\mathbf{C}_{1}-\mathbf{L} \Lambda\right) \mathbf{H}_{s}^{L}+\left(\mathbf{C}_{2}+\mathbf{R P}\right) \mathbf{H}_{s}^{R}\right]-\left[\left(\mathbf{C}_{1}^{*}-\mathbf{L}^{*} \Lambda\right) \mathbf{H}_{s}^{L}+\left(\mathbf{C}_{2}^{*}+\mathbf{R}^{*} \mathbf{P}\right) \mathbf{H}_{s}^{R}\right]\right\|^{2},
\end{aligned}
$$


where $\mathbf{C}_{1}, \mathbf{C}_{2}, \mathbf{L}, \mathbf{R}$ and $\mathbf{C}_{1}^{*}, \mathbf{C}_{2}^{*}, \mathbf{L}^{*}, \mathbf{R}^{*}$ are, respectively, the observed and estimated matrices of the left "centers" and right "centers," left spreads and right spreads of order $(n \times p)$ and the matrices $\mathbf{H}_{k}^{L}$ and $\mathbf{H}_{k}^{R}, s=1, \ldots, v$ are diagonal matrices with 0 and 1 diagonal elements, which allow us to describe suitable each and every vertex of the hyperrectangles (see Coppi et al. 2006a). The left and right spreads are differently weighted by means of the matrices $\boldsymbol{\Lambda}$ and $\mathbf{P}$. These are diagonal matrices whose elements are, respectively, $\lambda_{j}$ and $\rho_{j}$ that take values according to the left and right membership functions of the fuzzy variable at hand. More specifically, $\lambda_{j}=\int_{0}^{1} L_{j}^{-1}(\omega) \mathrm{d} \omega$ and $\rho_{j}=\int_{0}^{1} R_{j}^{-1}(\omega) \mathrm{d} \omega$, where $L_{j}$ and $R_{j}$ are, respectively, the left and right membership functions of the $j$-th fuzzy variable. These parameters take into account the variability of the membership function and reduce accordingly the influence of the spreads in the computation of the distance shown in Eq. (19), (for more details, see Coppi et al. 2006a). in which $\mathbf{A}_{C_{1}}, \mathbf{A}_{C_{2}}, \mathbf{A}_{L}$, and $\mathbf{A}_{R}$ are the component score matrices of order $(n \times \tilde{p})$ pertaining, respectively, to the left and right "centers," left spreads and right spreads, and $\tilde{p}$ is the number of extracted components. $\mathbf{F}(p \times \tilde{p})$ is the matrix of the component loadings that is assumed to be the same for the left and right "centers" and the (left and right) spreads. Finally, $\mathbf{E}_{C_{1}}, \mathbf{E}_{C_{2}}$ and $\mathbf{E}_{s}, s=1, \ldots, v$ are matrices of residuals of appropriate order.

The model shown in Eq. (21) aims at finding a compromise structure between centers and spreads: the vertices have the same component loadings, whereas the component scores take values around those pertaining to the left and right "centers." Once more, the model admits rotations of the obtained solution without affecting the model estimates.

By considering the least squares estimation procedure, the solution of the PCAF-LR model is based on the minimization of the distance shown in Eq. (20) with respect to $\mathbf{A}_{C_{1}}, \mathbf{A}_{C_{2}}, \mathbf{A}_{L}, \mathbf{A}_{R}$, and $\mathbf{F}$. For the iterative solution, see Coppi et al. (2006a).

In order to evaluate the model's fitting, Coppi et al. (2006a) proposed the following goodness of fit index:

$$
1-\frac{\left[\left\|\mathbf{C}_{1}-\mathbf{C}_{1}^{*}\right\|^{2}+\left\|\mathbf{C}_{2}-\mathbf{C}_{2}^{*}\right\|^{2}+\sum_{s=1}^{v}\left\|\left[\left(\mathbf{C}_{1}-\mathbf{L} \Lambda\right) \mathbf{H}_{s}^{L}+\left(\mathbf{C}_{2}+\mathbf{R P}\right) \mathbf{H}_{s}^{R}\right]-\left[\left(\mathbf{C}_{1}^{*}-\mathbf{L}^{*} \Lambda\right) \mathbf{H}_{s}^{L}+\left(\mathbf{C}_{2}^{*}+\mathbf{R}^{*} \mathbf{P}\right) \mathbf{H}_{s}^{R}\right]\right\|^{2}\right]}{\left\|\mathbf{C}_{1}\right\|^{2}+\left\|\mathbf{C}_{2}\right\|^{2}+\sum_{s=1}^{v}\left\|\left(\mathbf{C}_{1}-\mathbf{L} \Lambda\right) \mathbf{H}_{s}^{L}+\left(\mathbf{C}_{2}+\mathbf{R} \mathbf{P}\right) \mathbf{H}_{s}^{R}\right\|^{2}} .
$$

As shown by Coppi et al. (2006a), after some algebra, the distance shown in Eq. (19) can be simplified as follows:
High values of this index show that the PCAF-LR model fits the data very well.

$$
\begin{aligned}
& { }_{Y K} d_{L R}^{2}=\left(2^{J-1}+1\right)\left\|\mathbf{C}_{1}-\mathbf{C}_{1}^{*}\right\|^{2}+\left(2^{J-1}+1\right)\left\|\mathbf{C}_{2}-\mathbf{C}_{2}^{*}\right\|^{2}+2^{J-1}\left\|\mathbf{L} \Lambda-\mathbf{L}^{*} \Lambda\right\|^{2}+2^{J-1}\left\|\mathbf{R P}-\mathbf{R}^{*} \mathbf{P}\right\|^{2}+ \\
& -2^{J} \operatorname{tr}\left[\left(\mathbf{C}_{1}-\mathbf{C}_{1}^{*}\right)^{\prime}\left(\mathbf{L} \Lambda-\mathbf{L}^{*} \Lambda\right)\right]+2^{J} \operatorname{tr}\left[\left(\mathbf{C}_{2}-\mathbf{C}_{2}^{*}\right)^{\prime}\left(\mathbf{R P}-\mathbf{R}^{*} \mathbf{P}\right)\right] .
\end{aligned}
$$

4.4.1.2 Model The principal component analysis for Fuzzy LR data (PCAF-LR) adopts the following model:
To plot the objects into the low-dimensional space spanned by the orthonormal component loadings,

$\mathbf{C}_{1}=\mathbf{C}_{1}^{*}+\mathbf{E}_{C_{1}}$

$\mathbf{C}_{2}=\mathbf{C}_{2}^{*}+\mathbf{E}_{C_{2}}$

$\mathbf{C}_{1}^{*}=\mathbf{A}_{C_{1}} \mathbf{F}^{\prime}$

$\mathbf{C}_{2}^{*}=\mathbf{A}_{C_{2}} \mathbf{F}^{\prime}$

$\left(\mathbf{C}_{1}-\mathbf{L}\right) \mathbf{H}_{s}^{L}+\left(\mathbf{C}_{2}+\mathbf{R}\right) \mathbf{H}_{s}^{R}=\left(\mathbf{C}_{1}^{*}-\mathbf{L}^{*}\right) \mathbf{H}_{s}^{L}+\left(\mathbf{C}_{2}^{*}+\mathbf{R}^{*}\right) \mathbf{H}_{s}^{R}+\mathbf{E}_{s} s=1 \ldots, v$,

$\mathbf{L}^{*}=\mathbf{A}_{L} \mathbf{F}^{\prime}$

$\mathbf{R}^{*}=\mathbf{A}_{R} \mathbf{F}^{\prime}$ 
Coppi et al. (2006a, b, c, d) suggested a suitable plotting procedure.

\subsection{Multidimensional scaling for imprecise data}

In Multidimensional Scaling (MDS), the concept of distance/dissimilarity is involved in various steps, i.e., in the input data step (the input data are fuzzy distances/dissimilarities) and during the process of analysis. In classical MDS, the aim is to represent the data contained in a distance/dissimilarity (proximity) matrix onto a Euclidean space in which the distances between the "objects" approximate, in some sense, the observed distances/dissimilarities. This may happen in various situations. For instance, the distance/dissimilarity assessment may be formulated in linguistic terms (such as "very close," "close," "quite different," "different," "very different," and so on) or the assessment may be made by several judges in a quantitative way (thus causing an imprecision in any synthetic value chosen for summarizing the different distance/dissimilarity measures). In the above situations, the original data may be "fuzzified" so that to each pair of objects a distance/dissimilarity value is associated (Coppi 2003).

For instance, Hébert et al. (2006) point out the following examples of situations:

- "The dissimilarities may be directly elicited from human evaluators who have some difficulty in precisely quantifying the proximity of two objects. A fuzzy number rather than a real value may be more suitable to account for the vagueness of the evaluation."

- "The dissimilarities may be measured independently by several sensors, so that the available information concerning the dissimilarity between any two objects takes the form of an empirical distribution. One way to analyze such data is to describe the distribution by a fuzzy number computed from some fractiles of the distribution."

In the literature, some MDS methods in a fuzzy framework have been suggested. In Denoeux and Masson (2000) and Masson and Denoeux (2002), generalizations of Euclidean MDS to interval-valued and fuzzy data are suggested. Hébert et al. (2006) reviewed these generalizations and extended them to spherical MDS. In the same year, Groenen et al. (2006) suggested a MDS for interval dissimilarities based on iterative majorization. Masson and Denoeux (2002) suggested also a possibilistic version of the MDS by using in place of the least squares fitting a possibilistic fitting procedure.

\subsubsection{A representative example}

In the following, we show briefly the multidimensional scaling for fuzzy dissimilarities (MDSF) proposed by Masson and Denoeux (2002), which is characterized by the following steps:

1. input data and distance measure,

2. model.

4.5.1.1 Input data and distance measure The input data are represented by fuzzy distances/dissimilarities that, as explained before, may come from a linguistic evaluation by a single human subject or from the synthesis of responses from a panel of assessors.

Denoting by $\delta_{i i^{\prime}}$ the fuzzy distance/dissimilarity is measured between each pair of $i$-th and $i^{\prime}$-th object, $\left(i, i^{\prime}=1, \ldots ., n\right)$, and the input data matrix is constituted by $\boldsymbol{\Delta}=\left\{\delta_{i i^{\prime}}: i, i^{\prime}=1, \ldots, n\right\}$.

A LR membership function may be assigned to each fuzzy distance/dissimilarity, although this is not strictly necessary for applying the method. In fact, the basic idea of the method consists in focusing on the Euclidean representation of the objects, say $\Re^{\tilde{p}}$, where each $i$-th object is represented by a fuzzy region, say $R_{i}$. The modeling job is carried out with reference to this fuzzy representation.

Applying the extension principle (Zadeh 1975a, b, c), a fuzzy distance measure between two fuzzy regions $R_{i}$ and $R_{i}$, can be defined as follows:

$\mu_{d_{i^{\prime}}}(\omega)=\sup _{\mathbf{x}, \mathbf{y} \in \Re \tilde{R}} \min \left(\mu_{R_{i}}(\mathbf{x}), \mu_{R_{i^{\prime}}}(\mathbf{y})\right)$,

where the supremum is computed under the constraint $\omega=\|\mathbf{x}-\mathbf{y}\|$. If $R_{i}$ and $R_{i}$, are multidimensional fuzzy numbers, each $\alpha$-cut of $d_{i i^{\prime}}$ is a closed interval ${ }^{\alpha} d_{i i^{\prime}}=\left[{ }^{\alpha} d_{i i^{\prime}}^{-},{ }^{\alpha} d_{i i^{\prime}}^{+}\right]$, whose bounds are, respectively, the minimum and maximum distances between the $\alpha$-cuts of $R_{i}$ and $R_{i}$. Masson and Deneoux (2002) choose to represent each object by a fuzzy region whose $\alpha$-cuts are concentric hyperspheres of radii $r_{i}$ and center $c_{i}$, so that

${ }^{\alpha} d_{i i^{\prime}}^{-}=\max \left(0, d_{i i^{\prime}}^{*}-{ }^{\alpha} r_{i}-{ }^{\alpha} r_{i^{\prime}}\right)$

${ }^{\alpha} d_{i i^{\prime}}^{+}=d_{i i^{\prime}}^{*}+{ }^{\alpha} r_{i}+{ }^{\alpha} r_{i^{\prime}}$,

where $d_{i i^{\prime}}^{*}$ denotes the Euclidean distance between centers $c_{i}$ and $c_{i}$.

4.5.1.2 Model By adopting the least squares principle, the following steps characterize the model:

- choice of a set of $z$ predetermined $\alpha$-cuts with the convention: $1=\alpha_{1}>\ldots>\alpha_{s}>\ldots>\alpha_{z}=0$; 
- definition of the stress function to be minimized: $\sigma(\Re)=\sum_{s=1}^{z} \sum_{i<i^{\prime}}\left[\left({ }^{\alpha_{s}} d_{i i^{\prime}}^{-}-{ }^{\alpha_{s}} \delta_{i i^{\prime}}^{-}\right)^{2}+\left({ }^{\alpha_{s}} d_{i i^{\prime}}^{+}-{ }^{\alpha_{s}} \delta_{i i^{\prime}}^{+}\right)\right]$,

where $\Re$ indicates the set of fuzzy regions $R_{i}$.

The number of parameters of the model is $n(\tilde{p}+z)$ given by $n$ centers with $\tilde{p}$ coordinates, plus $n \times z$ radii $r_{i}$ (constrained by $z-1$ inequalities). Introducing the parameters ${ }^{\alpha_{s}} \eta_{i}$ such that $\alpha_{s} r_{i}=\sum_{h=1}^{s} \alpha_{h} \eta_{i}$ the above problem becomes an unconstrained non-linear optimization problem, which can be solved by means of an iterative gradient descent algorithm.

The amount of stress may also be used for judging the goodness of fit of the MDSF. The evaluation of the goodness of fit can be complemented graphically by the scree plot and the modified Shepard diagram (Hébert et al. 2006).

\subsection{Other exploratory multivariate methods for imprecise data}

Within an exploratory approach, there are other multivariate statistical methods for imprecise data. In particular, the following multivariate analysis can be considered:

- Correspondence analysis Loslever and Bouilland (1999) illustrated a fruitful marriage, to paraphrase the authors, between fuzzy sets and multiple correspondence analysis. Starting from a two-way contingency table where its elements are positive fuzzy numbers and considering the fuzzy eigenvalue concept, Theodorou et al. (2007) suggested a Correspondence Analysis for fuzzy data. In this context it is useful the concept of metric spaces for fuzzy sets (Diamond and Kloeden 1999) and the definition in a fuzzy manner of the so-called chi-square metric. Pinti et al. (2010) developed a tool in Matlab for multiple correspondence analysis of fuzzy coded data. Aşan and Greenacre (2011) using a fuzzy version of multiple correspondence analysis, suggested biplots of fuzzy coded data for visualizing them.

- Clusterwise regression analysis In considering a multivariate version of the Yank-Ko metric in the estimation process, D'Urso and Santoro (2006a, b) and D'Urso et al. (2010), in order to overcome the heterogeneity problem in fuzzy regression analysis of fuzzy data, proposed various methods for clusterwise regression analysis of various types of fuzzy data, in which the regression and clustering goals are taken into account simultaneously in a fuzzy framework.

- Classification and Regression trees To detect the number and location of change points of a fuzzy time series, Cappelli et al. (2013) employed a Yang-Ko distancebased deviation measure and its decomposition proper- ties (see D'Urso and Santoro 2006a, b) in the framework of the so-called Atheoretical Regression Trees (ART). Lertworaprachaya et al. (2014) suggested an intervalvalued fuzzy decision trees with optimal neighborhood perimeter Recently, Cappelli et al. (2015) suggested a regime change analysis of imprecise time series (i.e., interval-valued time series) based on regression trees. Useful references on decision trees in a fuzzy framework which can be considered for future studies are, e.g., Suarez and Lutsko (1999), Chiang and Hsu (2002), Olaru and Wehenkel (2003), Qin and Lawry (2005), Wang et al. (2008), Zeinalkani and Eftekhari (2014).

- Three-way analysis: in the last decades, increasing attention has also been paid to fuzzy clustering models for complex structures of fuzzy data. In particular, in a three-way framework, Coppi and D'Urso $(2002,2003)$ suggested fuzzy clustering models for fuzzy time-varying data arrays based on suitable metrics, respectively, an unweighted version of the distance shown in Eq. (6) for a particular case of LR fuzzy data, i.e., triangular fuzzy data, and a multivariate version of the Yang-Ko distance (Yang and Ko 1996). Other models in the same filed have been suggested by Coppi et al. (2004, 2006d). In the statistical area of three-way PCA for imprecise data, we point out the contributions by Giordani (2006, 2010).

- Discriminant analysis Following a non-parametric approach Colubi et al. (2011) proposed a discriminant analysis for fuzzy data obtained from a random experiment. References on fuzzy discriminant analysis useful for future works are, e.g., Watada et al. (1986), Tanaka et al. (1993), Chen et al. (1999), Lin and Chen (2004), $\mathrm{Wu}$ and Zhou (2006), Song et al. (2010), Shia et al. (2011), Heo and Gader (2011).

- Support vector machine for classification and regression Useful references in this field are, e.g., Hong and Hwang (2003), Celikyilmaz and Turksen (2007), Wu and Law (2010), Wu (2010), Batuwita and Palade (2010), Abe (2015).

- Strategies of analyses In many real situations when the structure of the imprecise information is more complex and the aims are multiple (e.g., data reduction with respect to objects and variables) it is necessary to adopt strategies of analysis in which all the previous multivariate methods for fuzzy data are suitably combined (e.g., principal component analysis and cluster analysis). In this connection, the previous methodological approaches can be suitably combined and integrated to account for the complexity of the empirical information.

- Hybrid analysis In recent years, several exploratory multivariate methods have been proposed in which the analyzed fuzzy variables are modeled within an inferential framework (fuzzy random variables). For instance, 
Table 1 Some references on real applications

\begin{tabular}{|c|c|}
\hline Methods & Examples of real applications \\
\hline Cluster analysis & $\begin{array}{l}\text { Behavior analysis (D’Urso et al. 2017); chemical analysis (D'Urso and Giordani 2006b; D'Urso and De Gio- } \\
\text { vanni 2014); consumers are social beings (D'Urso et al. 2015); e-health (D’Urso et al. 2013a); ecotoxicology } \\
\text { (D’Urso and De Giovanni 2014); environmental analysis (D'Urso et al. 2017); medicine (D'Urso and Giordani } \\
\text { 2006a); meteorology (D'Urso et al. 2015; D'Urso and Leski 2016); sensorial analysis (D'Urso and De Gio- } \\
\text { vanni 2014; Hung and Yang 2005); student performance (D'Urso and Giordani 2006a); tourism (D'Urso et al. } \\
\text { 2013b, 2015a, b, 2017) }\end{array}$ \\
\hline Self-organizing maps (SOMs) & $\begin{array}{l}\text { Meteorology (Hamdan and Hajjar 2011; Cabanes et al. 2013; D’Urso et al. 2014); satisfaction analysis (D'Urso } \\
\text { et al. 2014); sensorial evaluation (D’Urso et al. 2014); social opinion (D’Urso et al. 2014); telecommunication } \\
\text { (D’Urso and De Giovanni, 2011) }\end{array}$ \\
\hline Regression analysis & $\begin{array}{l}\text { Chemical analysis (D'Urso and Santoro 2006a, b); economics (Manski and Tamer 2002); environmental analysis } \\
\text { (Coppi et al. 2006a, b, c, d; D'Urso et al. 2011; D'Urso and Massari 2013); expert evaluation (D'Urso 2003; } \\
\text { D'Urso and Gastaldi 2000); finance (Gonzalez-Rivera and Lin 2013); human performance (D'Urso 2003); new } \\
\text { media opinion (D’Urso and Massari 2013); software reliability (D’Urso and Gastaldi 2002) }\end{array}$ \\
\hline Principal component analysis & $\begin{array}{l}\text { Behavioral analysis (Calcagnì et al. 2016); chemometrics (Giordani and Kiers 2004a, b; D’Urso and Giordani } \\
\text { 2004, 2005); decision making (Liu et al. 2014; 2015b); environmental analysis (Sarbu and Pop 2005; Pop et al. } \\
\text { 2009); face recognition analysis (Le-Rademacher and Billar 2012); satisfaction analysis (D’Urso and Giordani } \\
\text { 2005); sensorial analysis (Denœux and Masson 2004; Giordani and Kiers 2006); socioeconomics (Calcagnì } \\
\text { et al. 2016); social opinion (Coppi et al. 2006a, b, c, d) }\end{array}$ \\
\hline Multidimensional scaling & $\begin{array}{l}\text { Chemical analysis (Denoeux and Masson 2000); sensorial analysis (Masson and Denoeux 2002; Hébert et al. } \\
\text { 2006); sound perception (Groenen et al. 2006); speech signal (Denœux and Masson 2000) }\end{array}$ \\
\hline Correspondence analysis & $\begin{array}{l}\text { Medicine (Loslever and Bouilland 1999); meteorology (Aşan and Greenacre 2011); morphological evaluation } \\
\text { (Pinti et al. 2010); psychology (Loslever and Bouilland 1999) }\end{array}$ \\
\hline Clusterwise regression & $\begin{array}{l}\text { Expert evaluation (D'Urso et al. 2010); electricity consumption (D'Urso et al. 2010); environmental analysis } \\
\text { (D'Urso et al. 2010); tone perception analysis (D'Urso et al. 2006a, b) }\end{array}$ \\
\hline Regression trees & Environmental analysis (Cappelli et al. 2015); finance (Cappelli et al. 2013); meteorology (Cappelli et al. 2013) \\
\hline Three-way analysis & $\begin{array}{l}\text { Banking evaluation (Giordani 2010); oenology (Coppi and D’Urso 2002); tourism (Coppi and D’Urso, 2002); } \\
\text { web advertising (Coppi and D’Urso, 2003a, b; Giordani 2006) }\end{array}$ \\
\hline
\end{tabular}

Colubi et al. (2009) proposed a clustering method for fuzzy random variables based on the hierarchical clustering approach.

\section{Final remarks and future perspectives}

In this paper, we have shown the usefulness of fuzzy sets theory for exploratory multivariate statistical analysis when the empirical information is affected by uncertainty, imprecision, or vagueness (i.e., the data are imprecise). In this respect, we have focused our attentions on certain specific areas of this branch of statistics, i.e., cluster analysis, selforganizing maps, regression analysis, principal component analysis, and multidimensional scaling.

We have provided an outline of these methodological approaches within an overall framework, which is the basis of the general fuzzy approach to multivariate statistical analysis and presented a detailed review of the theoretical contributions in the various thematic areas.

The relevant impact of the various methodological approaches is also underscored by the numerous applications in many practical fields. References to some examples of real applications are shown in Table 1.

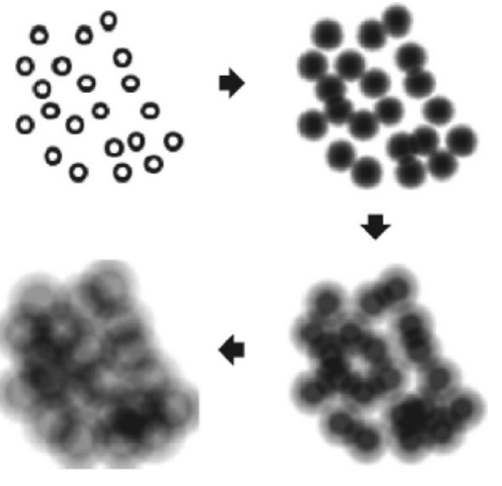

Fig. 3 Example of granulation of objects. (source Peters and Weber 2016)

As we have shown, in this field a lot has been done, but much more remains to be explored.

In the future, it could be stimulating to investigate in depth the fuzzy techniques for modeling the imprecision affecting the data in specific areas of statistics, such as multiple correspondence analysis, canonical correlation analysis, three-way data analysis (e.g., STATIS, Tucker, Dynamic Factor Analysis), decision trees, and support vector machine for classification and regression. 
In addition, since there are real situations in which the increasing level of complexity requires sophisticated tools to manage uncertainty, it would be interesting to develop exploratory multivariate methods based on extensions and derivatives of the fuzzy sets theory, such as the Type-2 fuzzy sets (Zadeh 1975a, b, c), the Intuitionistic fuzzy sets (Atanassov 1986), the Rough sets (Pawlak 1991), the Evidential theory (Dempster 1967; Shafer 1976), and the Shadowed sets (Pedrycz 1998).

In this connection, to manage the imprecision affecting the empirical information in data analysis, it could be useful to utilize the techniques of granular computing (Bargiela and Pedrycz 2003; Pedrycz 2007; Pedrycz et al. 2008) in the exploratory multivariate framework. In fact, as Zadeh (1997) wrote "fuzzy information granulation underlies the remarkable human ability to make rational decisions in an environment of imprecision, partial knowledge, partial certainty and partial truth." Notice that in a granular framework, "by information granules one regards a collection of elements drawn together by their closeness (resemblance proximity, functionality, etc.) articulated in terms of some useful spatial, temporal, or functional relationships. Subsequently, Granular Computing is about representing, constructing, and processing information granules" (Pedrycz et al. 2015a). In this respect, e.g., in the clustering framework, in terms of granular computing, a cluster can be interpreted as an information granule that presents its objects on a coarser and more granular level (Gacek and Pedrycz 2015). In Fig. 3 (drawn by Peters and Weber 2016), we show an example of granulation of objects. Useful references in this field are, e.g., Pedrycz and Bagiela 2002; Sanchez et al. 2014; Gacek and Pedrycz 2015; Pedrycz et al. 2015b; Peters and Weber 2016; Lingras et al. 2016; Dubois and Prade 2016. In addition to cluster analysis, other areas of the exploratory multivariate statistics can benefit from the use of granular computing tools, such as regression analysis, principal component analysis, and so on. In particular, for regression analysis, we draw attention to the following contributions: Roychowdhury and Pedrycz 2002; Peters 2011; Peters and Lacic 2012; Cimino et al. 2014. A useful reference on granular fuzzy data analysis is Pedrycz (2015).

Notice that, in a granular perspective, in this paper, we explored only a particular case of granular statistical methods, i.e., the fuzzy statistical methods-fuzzy clustering, fuzzy SOMs, fuzzy regression, fuzzy PCA, fuzzy MDS, fuzzy correspondence analysis, and so on. Since there are other well-known formal settings where information granules can be expressed and processed (Pedrycz et al. 2015a)—such as sets (intervals), shadowed sets, probability-oriented information granules, and rough sets-in future it will be interesting to explore other typologies of granular exploratory multivariate methods. In particular, in a clustering framework, it will be interesting to explore, e.g., shadowed sets-based clustering, rough clustering, intuitionistic clustering, and type-2 fuzzy clustering; in a regression framework, it will be useful to review, rough regression, interval-valued regression, and so on (Pedrycz 2015).

In conclusion, when using a hybrid approach (statistical modeling based on simultaneously exploratory and inferential tools), it could be particularly interesting to study exploratory multivariate methods for imprecise data in which inferential tools-such as likelihood function defined in a fuzzy framework, fuzzy extension of the EM algorithm or statistical test procedures-are considered (see, e.g., Colubi et al. 2009; Quost and Denoeux 2016).

All the previous methodological approaches can be explored individually and simultaneously from the perspective of analysis strategy. In this way, complex structures and very large amounts of imprecise data (big imprecise data) can be fruitfully analyzed using a synergic combination of the various methods.

Fields that could benefit from the application of these methodological approaches are, for instance, web analytics, social network, econophysics, neuroeconomics, and finance.

Acknowledgements The author wishes to thank the editors and referees for their useful comments and suggestions which helped to improve the quality and presentation of this paper.

\section{References}

Abe S (2015) Fuzzy support vector machines for multilabel classification. Pattern Recognit 48:2110-2117

Akdag H, Kalayci T, Karagoz S, Zulfikar H, Giz D (2014) The evaluation of hospital service quality by fuzzy MCDM. Appl Soft Comput 23:239-248

Aşan Z, Greenacre M (2011) Biplots of fuzzy coded data. Fuzzy Sets Syst 183: 57-71

Atanassov K (1986) Intuitionistic fuzzy sets. Fuzzy Sets Syst 20: 87-96

Auephanwiriyakul S, Keller JM (2002) Analysis and efficient implementation of a linguistic fuzzy c-means. IEEE Trans Fuzzy Syst 10:563-582

Baklouti R, Mansouri M, Nounou M, Nounou H, Hamida AB (2016) Iterated robust kernel fuzzy principal component analysis and application to fault detection. J Comput Sci 15:34-49

Bargiela A, Pedrycz W (2003) Granular computing: an introduction. Kluwer Academic Publishers, Boston

Barni M, Cappellini V, Mecocci A (1996) Comments on 'a possibilistic approach to clustering'. IEEE Trans Fuzzy Syst 4:393-396

Batuwita R, Palade V (2010) FSVM-CIL: Fuzzy Support Vector Machines for Class Imbalance Learning. IEEE Trans Fuzzy Syst 18:558-571

Bloch I (1999) On fuzzy distances and their use in image processing under imprecision. Pattern Recognit 32:1873-1895 
Bock HH (1999) Clustering methods and Kohonen maps for symbolic data. J Jpn Soc Comput Stat 15:217-229

Bock HH (2008) Visualizing symbolic data by Kohonen maps. In: Diday E, Noihome-Fraiture M (eds), Symbolic Data Analysis and the SODAS Software, Wiley, 205-234

Butkiewicz BS (2005) Robust Fuzzy clustering with Fuzzy data. Proceedings of advances in web intelligence, Third International Atlantic Web Intelligence Conference, AWIC 2005, Lecture Notes in Computer Science, Springer, 352, 76-82

Cabanes G, Bennani Y, Destenay R, Hardy A (2013) A new topological clustering algorithm for interval data. Pattern Recognit 46:3030-3039

Calcagnì A, Lombardi L, Pascali E (2016) A dimension reduction technique for two-mode non-convex fuzzy data. Soft Comput 20:749-762

Campello R, Hruschka E (2006) A fuzzy extension of the silhouette width criterion for cluster analysis. Fuzzy Sets Syst 157:2858-2875

Cappelli C, D'Urso P, Di Iorio F (2013) Change point analysis for imprecise time series. Fuzzy Sets Syst 225:23-38

Cappelli C, D’Urso P, Di Iorio F (2015) Regime change analysis of interval-valued time series with an application to PM10. Chemom Intell Lab Syst 146:337-346

Celikyilmaz A, Turksen IB (2007) Fuzzy functions with support vector machines. Inf Sci 177:5163-5177

Celminš A (1987) Multidimensional least-squares fitting of fuzzy models. Math Model 9:669-690

Celminš A (1991) A practical approach to nonlinear fuzzy regression, SIAM. J Sci Stat Comput 12(3):521-546

Chang YH, Ayyub BM (2001) Fuzzy regression methods-a comparative assessment. Fuzzy Sets Syst 119:187-203

Chang PT, Lee ES (1996) A generalized fuzzy weighted leastsquares regression. Fuzzy Sets Syst 82:289-298

Chen S, Yeh M, Hsiao P (1995) A comparison of similarity measures of fuzzy values. Fuzzy Sets Syst 72:79-89

Chen Z-P, Jiang J-H, Liang Y-Z, Yu R-Q (1999) Fuzzy linear discriminant analysis for chemical data sets. Chemom Intell Lab Syst 45:295-302

Chen D, Hung W, Yang M (2010) A batch version of the SOM for symbolic data. In: 2010 Sixth International Conference on Natural Computation (ICNC), vol. 1, IEEE, 2010, 1-5

Chiang I-J, Hsu JY (2002) Fuzzy classification trees for data analysis. Fuzzy Sets Syst 130:87-99

Cimino MGCA, Lazzerini B, Marcelloni F, Pedrycz W (2014) Genetic interval neural networks for granular data regression. Inf Sci 257:313-330

Colubi A, Gonzales Rodriguez G, D’Urso P, Montenegro M (2009) Multi-sample test-based clustering for Fuzzy random variables. Int J Approx Reas 50(5):721-731

Colubi A, Gonzalez-Rodriguez G, Gil MA, Trutschnig W (2011) Nonparametric criteria for supervised classification of fuzzy data. Int J Approx Reason 52:1272-1282

Coppi R (2002) A theoretical framework for Data Mining: the "Informational Paradigm". Compu Stat Data Anal 38:501-515

Coppi R (2003) The fuzzy approach to multivariate statistical analysis, Technical report, Dipartimento di Statistica, Probabilità e Statistiche Applicate, Sapienza Università di Roma, n. 11

Coppi R (2008) Management of uncertainty in statistical reasoning: the case of regression analysis. Int J Approx Reason 47:284-305

Coppi R, D’Urso P (2002) Fuzzy K-means clustering models for triangular fuzzy time trajectories. Stat Methods Appl 11(1):21-40

Coppi R, D'Urso P (2003a) Three-way Fuzzy clustering models for LR fuzzy time trajectories. Comput Stat Data Anal 43:149-177
Coppi R, D’Urso P (2003b) Regression analysis with Fuzzy informational paradigm: a least-squares approach using membership function information. Int J Pure Appl Math 8:279-306

Coppi R, D’Urso P, Giordani P (2004) Informational Paradigm and Entropy-Based Dynamic Clustering in a Complete Fuzzy Framework, in Soft Methodology in Random Information Systems (eds. Angeles Gil, M., Lopez-Diaz, M.C., Grzegorzewski, P.) (in Advances in Soft Computing), 2nd International Conference on Soft Methods in Probability and Statistics (SMPS2004), September, 2-4, Oviedo (Asturias) Spain, 463-470, SpringerVerlag Heidelberg, 2004

Coppi R, Giordani P, D’Urso P (2006a) Component models for Fuzzy data. Psychometrika 71:733-761

Coppi R, Gil MA, Kiers, HAL (2006b) The fuzzy approach to statistical analysis. Comput Stat Data Anal 51:1-14

Coppi R, D’Urso P, Giordani P, Santoro A (2006c) Least squares estimation of a linear regression model with LR Fuzzy response. Comput Stat Data Anal 51:267-286

Coppi R, D’Urso P, Giordani P (2006d) Fuzzy K-Medoids Clustering Models for Fuzzy Multivariate Time Trajectories, COMPSTAT 2006, Rome, 28 August-1 September, 2006, Proceeding in Computational Statistics (eds. A. Rizzi, M. Vichi), PhysicaVerlag, 17-29

Coppi R, D’Urso P, Giordani P (2012) Fuzzy and possibilistic clustering models for fuzzy data. Comput Stat Data Anal 56:915-927

D'Urso P (2003) Linear regression analysis for Fuzzy/crisp input and Fuzzy/crisp output data. Comput Stat Data Anal 42(1-2):47-72

D'Urso P (2007) Fuzzy clustering of Fuzzy data, in "Advances in Fuzzy Clustering and Its Applications" (eds. J.V. de Oliveira, W. Pedrycz). J Wiley Sons 155-192:2007

D’Urso P (2015) Fuzzy clustering. In: Hennig C, Meila M, Murtagh F, Rocci R (eds) Handbook of cluster analysis. Chapman \& Hall, Boca Raton, 545-573

D'Urso P, De Giovanni, L (2011) Midpoint radius self-organizing maps for interval-valued data with telecommunications application. Appl Soft Comput 11:3877-3886

D’Urso P, De Giovanni L (2014) Robust clustering of imprecise data. Chemom Intell Lab Syst 136:58-80

D’Urso P, Gastaldi T (2000) A least-squares approach to Fuzzy linear regression analysis. Comput Stat Data Anal 34:427-440

D'Urso P, Gastaldi T (2002) An "orderwise" polynomial regression procedure for Fuzzy data. Fuzzy Sets Syst 130(1):1-19

D’Urso P, Giordani P (2004) A least squares approach to principal component analysis for interval valued data. Chemom Intell Lab Syst 70:179-192

D’Urso P, Giordani P (2005) A possibilistic approach to latent component analysis for symmetric fuzzy data. Fuzzy Sets Syst 150:285-305

D'Urso P, Giordani P (2006a) A weighted Fuzzy c-means clustering model for Fuzzy data. Comput Stat Data Anal 50(6):1496-1523

D’Urso P, Giordani P (2006b) A robust Fuzzy k-means clustering model for interval valued data. Comput Stat 21:251-269

D’Urso P, Leski J (2016) Fuzzy C-ordered medoids clustering of interval-valued data. Pattern Recognit 58:9-67

D’Urso P, Massari R (2013) Weighted least squares and least median squares estimation for the fuzzy linear regression analysis. Metron 71:279-306

D’Urso P, Santoro A (2006a) Goodness of fit and variable selection in the Fuzzy multiple linear regression. Fuzzy Sets Syst 157:2627-2647

D’Urso P, Santoro A (2006b) Fuzzy clusterwise regression analysis with symmetrical Fuzzy output variable. Comput Stat Data Anal 51:287-313

D’Urso P, Massari R, Santoro A (2010) A class of Fuzzy clusterwise regression models. Inform Sci 180:4737-4762 
D’Urso P, Massari R, Santoro A (2011) Robust Fuzzy regression analysis. Inform Sci 181:4154-4174

D’Urso P, De Giovanni L, Spagnoletti P (2013a) A Fuzzy taxonomy for e-health projects. Int J Mach Learn Cybern 4:487-504

D’Urso P, De Giovanni L, Disegna M, Massari R (2013b) Bagged clustering and its application to tourism market segmentation. Expert Systems Appl 40:4944-4956

D’Urso P, De Giovanni L, Massari R (2014) Self-organizing maps for imprecise data. Fuzzy Sets Syst 237:63-89

D’Urso P, Disegna M, Massari R, Prayag G (2015a) Bagged fuzzy clustering for fuzzy data: an application to a tourism market, Knowl-Based Syst 73:335-346

D’Urso P, De Giovanni L, Massari R (2015b) Trimmed fuzzy clustering for interval-valued data. ADAC 9:21-40

D’Urso P, De Giovanni L, Massari R, Cappelli C (2017) Exponential distance-based fuzzy clustering for interval-valued data. Fuzzy Optim Decis Mak 16:51-70

de Carvalho FDA (2007) Fuzzy c-means clustering methods for symbolic interval data. Pattern Recognit Lett 28:423-437

de Sousa RMCR, De Carvalho FAT (2004) Clustering of interval data based on city-block distances. Pattern Recognit Lett 25:353-365

de Carvalho FDA, de Souza RMCR (2010) Unsupervised pattern recognition models for mixed feature-type symbolic data. Pattern Recognit Lett 31:430-443

de Carvalho FDA, Lechevallier Y (2009a) Dynamic clustering of interval-valued data based on adaptive quadratic distances. IEEE Trans Syst Man Cybern Part A: Syst Hum 39:1295-1306

de Carvalho FDA, Lechevallier Y (2009b) Partitional clustering algorithms for symbolic interval data based on single adaptive distances. Pattern Recognit 42:1223-1236

de Carvalho FDA, Tenorio C (2010) Fuzzy k-means clustering algorithms for interval valued data based on adaptive quadratic distances. Fuzzy Sets Syst 161:2978-2999

De Luca A, Termini S (1972) A definition of non-probabilistic entropy in the setting of fuzzy set theory. Inf Control 20:301-312

de Carvalho FDA, Brito P, Bock HH (2006a) Dynamic clustering for interval data based on L2 distance. Comput Stat 21:231-250

de Carvalho FDA, de Souza RMCR, Chavent M, Lechevallier Y (2006b) Adaptive Hausdorff distances and dynamic clustering of symbolic interval data. Pattern Recognit Lett 27:167-179

de la Rosa de Saa S, Gil MA, Gonzalez-Rodriguez G, Lopez MT, Lubiano MA (2015) Fuzzy rating scale-based questionnaires and their statistical analysis. IEEE Trans Fuzzy Syst 23:111-126

Dempster AP (1967) Upper and lower probabilities induced by a multivalued mapping. Ann Math Stat 38:325-339

Denoeux T, Masson M-H (2000) Multidimensional scaling of interval-valued dissimilarity data. Pattern Recognit Lett 21:83-92

Denœux T, Masson M (2004) Principal component analysis of fuzzy data using autoassociative neural networks. IEEE Trans Fuzzy Syst 12:336-349

Denœux T, Masson M (2007) Dimensionality reduction and visualization of interval and fuzzy data: a survey. Bulletin of the International Statistical Institute LXII (Proceedings of the 56th session of the International Statistical Institute (ISI '07)), 627-634, Lisboa, Portugal, August 2007

Diamond P (1988) Fuzzy least squares. Inform Sci 46:141-157

Diamond P, Kloeden P (1999) Metric spaces of fuzzy sets. Fuzzy Sets Syst 100:63-71

Diamond P, Tanaka H (1998) Fuzzy regression analysis. In: Slowinski $\mathrm{R}$ (ed) Fuzzy sets in decision analysis, operations research and statistics. Kluwer Academic Publishers, Boston, pp 349-387

Douzal-Chouakria A, Billard L, Diday E (2011) Principal component analysis for interval-valued observations. Stat Anal Data Min 4:229-246
Dubois D, Prade H (1988) Possibility theory, Plenum Press, New York

Dubois D, Prade H (2016) Bridging gaps between several forms of granular computing, Granular. Computing 1:1115-1126

El Golli A, Conan-Guez B, Rossi F (2004) Self-organizing maps and symbolic data. JSDA Electron J Symbol Data Anal 2

El-Sonbaty Y, Ismail MA (1998) Fuzzy clustering for symbolic data. IEEE Trans Fuzzy Syst 6:195-204

Estrella FJ, Espinilla M, Herrera V, Martinez L (2014) FLINTSTONES: a fuzzy linguistic decision tools enhancement suite based on the 2-tuple linguistic model and extensions. Inf Sci 280:152-170

Frigui H, Krishnapuram R (1996) A robust algorithm for automatic extraction of an unknown number of clusters from noisy data. Pattern Recognit Lett 17(12):1223-1232

Gacek A, Pedrycz W (2015) Clustering granular data and their characterization with information granules of higher type. IEEE Trans Fuzzy Syst 23(4):850-860

Garcia-Galan S, Prado RP, Exposito, JEMN (2015) Rules discovery in fuzzy classifier systems with PSO for scheduling in grid computational infrastructures. Appl Soft Comput 29:424-435

Giordani P (2006) Two-and three-way component models for LR fuzzy data in a possibilistic framework. Fuzzy Sets Syst 157:2648-2664

Giordani P (2010) Three-way analysis of imprecise data. J Multivar Anal 101:568-582

Giordani P, Kiers, HAL (2004a) Principal Component Analysis of symmetric fuzzy data. Comput Stat Data Anal 45:519-548

Giordani P, Kiers, HAL (2004b) Three-way component analysis of interval valued data. J Chemometr 18:253-264

Giordani P, Kiers, HAL (2006) A comparison of three methods for principal component analysis of fuzzy interval data. Comput Stat Data Analysis 51:379-397

Gonzalez-Rivera G, Lin W (2013) Constrained regression for interval-valued data. J Bus Econ Stat 31(4):473-490

Groenen PJF, Winsberg S, Rodrìguez O, Diday E (2006) I-Scal: Multidimensional scaling of interval dissimilarities. Comput Stat Data Anal 51:360-378

Guru DS, Kiranagi BB, Nagabhushan P (2004) Multivalued type proximity measure and concept of mutual similarity value useful for clustering symbolic patterns. Pattern Recognit Lett 25:1203-1213

Hajjar C, Hamdan H (2011a) Self-organizing map based on L2 distance for interval-valued Data, IEEE International Symposium on Applied Computational Intelligence and Informatics, Timisoara, Romania, 19-21 may, 317-322

Hajjar C, Hamdan H (2011b) Self-organizing map based on Hausdorff distance for interval-valued data, IEEE International Conference on Systems, Man, and Cybernetics, Anchorage, Alaska, 9-12 october, $1747-1752$

Hajjar C, Hamdan H (2012) Self-organizing map based on city-block distance for interval-valued data. In: Aiguier M, Bretaudeau F, Krob D (eds), Complex systems design and management, Springer, Heidelburg, 281-292

Hajjar C, Hamdan H (2013) Interval data clustering using self-organizing maps based on adaptive Mahalanobis distances. Neural Netw 46:124-132

Hamdan H, Hajjar C (2011) A neural networks approach to intervalvalued data clustering. Application to Lebanese meteorological stations data, IEEE Workshop on Signal Processing Systems, Beirut, Lebanon, 4-7 October, 373-378

Hamdan H, Hajjar C (2012) Kohonen Neural Networks for Intervalvalued Data Clustering. Int J Adv Comput Sci 2:412-419

Hardy A, Kasaro N (2009) A new clustering method for interval data. Math Sci Hum 187:79-91 
Hathaway RJ, Bezdek JC, Pedrycz W (1996) A parametric model for fusing heterogeneous fuzzy data. IEEE Trans Fuzzy Syst 4:1277-1282

Hébert P-A, Denoeux T, Masson M-H (2006) Fuzzy multidimensional scaling. Comput Stat Data Anal 51:335-359

Heo G, Gader P (2011) Robust kernel discriminant analysis using fuzzy memberships. Patter Recognit 44:716-723

Heo G, Gader P, Frigui H (2009) RKF-PCA: Robust kernel fuzzy PCA. Neural Netw 22:642-650

Herrera F, Herrera-Viedma E, Verdegay JL (1998) Choice processes for non-homogeneous group decision making in linguistic setting. Fuzzy Sets Syst 94:287-308

Herrera F, Herrera-Viedma E, Martinez L (2008) A fuzzy linguistic methodology to deal with unbalanced linguistic term sets. IEEE T Fuzzy Syst 16:354-370

Hesketh T, Hesketh B (1994) Computerized fuzzy ratings: the concept of a fuzzy class. Behav Res Meth Ins C 26, 272-277

Hesketh T, Pryor R, Hesketh B (1988) An application of a computerized fuzzy graphic rating scale to the psychological measurement of individual differences. Int J Man Mach Stud 29:21-35

Hesketh B, Griffin B, Loh V (2011) A future-oriented retirement transition adjustment framework. J Vocat Behav 79:303-314

Honda K, Ichihashi H (2006) Fuzzy local independent component analysis with external criteria and its application to knowledge discovery in databases. Int J Approx Reason 42:159-173

Honda K, Notsu A, Ichihashi H (2010) Fuzzy PCA-guided robust k-means clustering. IEEE Trans Fuzzy Syst 18:67-79

Hong DH, Hwang C (2003) Support vector fuzzy regression machines. Fuzzy Sets Syst 138:271-281

Hung WL, Yang MS, (2005) Fuzzy clustering on LR-type fuzzy numbers with an application in Taiwanese tea evaluation. Fuzzy Sets Syst 150:561-577

Hung WL, Yang MS, Lee ES (2010) A robust clustering procedure for fuzzy data. Comput Math Appl 60:151-165

Kao C-H, Nakano J, Shieh S-H, Tien Y-J, Wu H-M, Yang C-K, Chen C-H (2014) Exploratory data analysis of interval-valued symbolic data with matrix visualization. Comput Stat Data Anal 79:14-29

Kaufman L, Rousseeuw PJ (1990). Finding groups in data: an introduction to cluster analysis. New York, Wiley

Kim KJ, Moskovitz H, Koksalan M (1996) Fuzzy versus statistical linear regression. Eur J Oper Res 92:417-434

Klement E, Puri M, Ralescu D (1986) Limit theorems for fuzzy random variables. Proc Roy Soc London Ser A 407:171-182

Kohonen T, (1995), Self-organizing maps, Springer Verlag, Berlin

Krishnapuram R, Keller J (1996) The possibilistic c-means algorithm: insights and recommendations. IEEE Trans Fuzzy Syst 4:385-393

Kruse R, Meyer KD (1987) Statistics with Vague Data. Kluwer, Dordrecht

Kwakernaak H (1978) Fuzzy random variables-I. Inf Sci 15:1-29

Kwakernaak H (1979) Fuzzy random variables-II. Inf Sci $17: 253-278$

Lalla M, Facchinetti G, Mastroleo G (2008) Vagueness evaluation of the crisp output in a fuzzy inference system. Fuzzy Sets Syst 159:3297-3312

Le-Rademacher J, Billard L (2012) Symbolic covariance principal component analysis and visualization for interval-valued data. $\mathrm{J}$ Comput Gr Stat 21:413-432

Lertworaprachaya Y, Yang Y, John R (2014) Interval-valued fuzzy decision trees with optimal neighbourhood perimeter. Appl Soft Comput 24:851-866

Li Q (2013) A novel Likert scale based on fuzzy sets theory. Expert Syst Appl 40:1609-1618

Lin C-C, Chen A-P (2004) Fuzzy discriminant analysis with outlier detection by genetic algorithm. Comput Oper Res 31:877-888
Lingras P, Haider F, Triff M (2016) Granular meta-clustering based on hierarchical, network, and temporal connections. Granul Comput 1:71-92

Liu B, Chen Y, Shen Y, Sun H, Xu X (2014) A complex multi-attribute large-group decision making method based on the intervalvalued intuitionistic fuzzy principal component analysis model. Soft Comput 18:2149-2160

Liu R, Cui L, Zeng G, Wu H, Wang C, Yan S, Yan B (2015a) Applying the fuzzy SERVQUAL method to measure the service quality in certification and inspection industry. Appl Soft Comput 26:508-512

Liu B, Shen Y, Zhang W, Chen X, Wang X (2015b) An interval-valued intuitionistic fuzzy principal component analysis modelbased method for complex multi-attribute large-group decisionmaking. Eur J Oper Res 245:209-225

Loslever P, Bouilland S (1999) Marriage of fuzzy sets and multiple correspondence analysis: examples with subjective interval data and biomedical signals. Fuzzy Sets Syst 107:255-275

Lowen R, Peeters W (1998) Distances between fuzzy sets representing grey level images. Fuzzy Sets Syst 99:135-149

Lubiano MA, de la Rosa de Sàa S, Montenegro M, Sinova B, Gil MA (2016a) Descriptive analysis of responses to items in questionnaires. Why not using a fuzzy rating scale. Inf Sci 360:131-148

Lubiano MA, Montenegro M, Sinova B, de la Rosa de Sàa S, Gil MA (2016b) Hypothesis testing for means in connection with fuzzy rating scale-based data: algorithms and applications. Eur J Oper Res 251:918-929

Ma M, Friedman M, Kandel A (1997) General fuzzy least squares. Fuzzy Sets Syst 88:107-118

Manski CF, Tamer E (2002) Inference on regressions with interval data on a regressor or outcome. Econometrica 70:519-546

Massanet S, Riera JV, Torrens J, Herrera-Viedma E (2014) A new linguistic computational model based on discrete fuzzy numbers for computing with words. Inf Sci 258:277-290

Masson M-H, Denoeux T (2002) Multidimensional scaling of fuzzy dissimilarity data. Fuzzy Sets Syst 128:339-352

Matsui T, Takeya M (1994) Structural analysis method for fuzzy rating scale data using fuzzy integration, in: Proceedings of the 1994 IEEE International Conference on Systems, Man and Cybernetics, 1, 493-500

Olaru C, Wehenkel L (2003) A complete fuzzy decision trees technique. Fuzzy Sets Syst 138:221-254

Pappis C, Karacapilidis N (1993) A comparative assessment of measures of similarity of fuzzy values. Fuzzy Sets Syst $56: 171-174$

Pawlak Z (1991) Rough sets, theoretical aspects of reasoning about data. Kluwer Academic, Dordrecht

Pedrycz W (1989) A fuzzy cognitive structure for pattern recognition. Pattern Recognit Lett 9:305-313

Pedrycz W (1998) Shadowed sets: representing and processing fuzzy sets. IEEE Trans Syst Man Cybern Part B: Cybern 28:103-109

Pedrycz W (2007) Granular computing - the emerging paradigm. J Uncertain Syst 1:38-61

Pedrycz W (2013) Granular computing. Analysis and design of intelligent systems. CRC Press, Taylor and Francis Group, Boca Raton

Pedrycz W (2015) From fuzzy data analysis and fuzzy regression to granular fuzzy data analysis. Fuzzy Sets Syst 274:12-17

Pedrycz W, Bagiela A (2002) Granular clustering: a granular signature of data. IEEE Trans Syst Man Cybern Part B: Cybern 32:212-224

Pedrycz W, Bezdek JC, Hathaway RJ, Rogers GW (1998) Two nonparametric models for fusing heterogeneous fuzzy data. IEEE Trans Fuzzy Syst 6(3):411-425 
Pedrycz W, Skowron A, Kreinovich V (eds) (2008) Handbook of granular computing. Wiley, Chichester

Pedrycz W, Succi G, Sillitti A, Iljazi J (2015a) Data description: a general framework of information granules. Knowl-Based Syst 80:98-108

Pedrycz W, Al-Hmouz R, Balamash AS, Morfeq A (2015b) Hierarchical granular clustering: an emergence of information granules of higher order. IEEE Trans Fuzzy Syst 23:2270-2283

Peters, G. (2011) Granular box regression. IEEE Transac Fuzzy Syst 19:1141-1152

Peters G, Lacic Z (2012) Tackling outliers in granular box regression. Inf Sci 212:44-56

Peters G, Weber R (2016) DCC: a framework for dynamic granular clustering. Granul Comput 1:1-11

Pinti A, Rambaud F, Griffon J-L, Ahmed AT (2010) A tool developed in Matlab for multiple correspondence analysis of fuzzy coded data sets: application to morphometric skull data. Comput Methods Programs Biomed 98:66-75

Pop HF, Einax JW, Sârbu C (2009) Classical and fuzzy principal component analysis of some environmental samples concerning the pollution with heavy metals. Chemom Intell Lab Syst 97:25-32

Puri ML, Ralescu DA (1986) Fuzzy random variables. J Math Anal Appl 114:409-422

Qin Z, Lawry J (2005) Decision tree learning with fuzzy labels. Inf Sci 172:91-129

Quost B, Denoeux T (2016) Clustering and classification of fuzzy data using the fuzzy EM algorithm. Fuzzy Sets Syst 286:134-156

Rezaee MJ, Moini A (2013) Reduction method based on fuzzy principal component analysis in multi-objective possibilistic programming. Int J Adv Manuf Technol 67:823-831

Roychowdhury S, Pedrycz W (2002) Modeling temporal functions with granular regression and fuzzy rules. Fuzzy Sets Syst 126:377-387

Sanchez MA, Castillo O, Castro JR, Melin P (2014) Fuzzy granular gravitational clustering algorithm for multivariate data. Inf Sci 279:498-511

Sarbu C, Pop HF (2005) Principal component analysis versus fuzzy principal component analysis. A case study: the quality of Danube water (1985-1996). Talanta 65:1215-1220

Sato M, Sato Y, (1995) Fuzzy clustering model for fuzzy data. Proc IEEE 4:2123-2128

Shafer G (1976) A Mathematical Theory of Evidence, Princeton University Press, Princeton

Shia B-C, Zhu J, Fang K, Ma S (2011) Fuzzy canonical discriminant analysis: theory and practice. Commun Stat-Simul Comput 40:1526-1539

Song X-N, Zheng Y-J, Wu X-J, Yang X-J, Yang J-Y (2010) A complete fuzzy discriminant analysis approach for face recognition. Appl Soft Comput 10:208-214

Suarez A, Lutsko JF (1999) Globally optimal fuzzy decision trees for classification and regression. IEEE Trans Pattern Anal Mach Intell 21:1297-1311

Takata O, Miyamoto S, Umayahara K (2001) Fuzzy clustering of data with uncertainties using minimum and maximum distances based on L1 metric, Proceedings of Joint 9th IFSA World Congress and 20th NAFIPS International Conference, July 25-28, 2001, Vancouver, British Columbia, Canada, 2511-2516

Takemura K (1999) A fuzzy linear regression analysis for fuzzy input-output data using the least squares method under linear constraints and its application to fuzzy rating data. J Adv Comput Intel Intel Inf 3:36-41

Takemura K (2007) Ambiguous comparative judgment: fuzzy set model and data analysis. Jpn Psychol Res 49:148-156

Takemura K (2012) Ambiguity and social judgment: fuzzy set model and data analysis. In: Dadios EP (ed), Fuzzy logic—algorithms, techniques and implementations. InTech-Open Access Publisher, 3-24

Tanaka H, Watada J, (1988) Possibilistic linear systems and their application to the linear regression model. Fuzzy Sets Syst 27:275-289

Tanaka H, Uejima S, Asai K (1982) Linear regression analysis with fuzzy model. IEEE Trans Syst Man Cybernet 12:903-907

Tanaka H, Ishibuchi H, Hayashi I (1993) Identification method of possibility distributions and its application to discriminant analysis. Fuzzy Sets Syst 58:41-50

Tavana M, Caprio DD, Santos-Arteaga FJ (2015) A bilateral exchange model: the paradox of quantifying the linguistic values of qualitative characteristics. Inf Sci 296:201-218

Tejeda-Lorente A, Porcel C, Peis V, Sanz R, Herrera-Viedma E (2014) A quality based recommender system to disseminate information in a university digital library. Inf Sci 261:52-69

Tejeda-Lorente A, Bernabe-Moreno J, Porcel C, Galindo-Moreno P, Herrera-Viedma E (2015) A dynamic recommender system as reinforcement for personalized education by a fuzzly linguistic web system. Procedia Comput Sci 55:1143-1150

Theodorou Y, Drossos C, Alevizos P (2007) Correspondence analysis with fuzzy data: the fuzzy eigenvalue problem. Fuzzy Sets Syst 158:704-721

Tong RM, Bonissone PP (1980) A linguistic approach to decision making with fuzzy sets. IEEE Trans Syst Man Cybern 10:716-723

Villacorta PJ, Masegosa AD, Castellanos D, Lamata MT (2014) A new fuzzy linguistic approach to qualitative cross impact analysis. Appl Soft Comput 24:19-30

Wang W (1997) New similarity measures on fuzzy sets and on elements. Fuzzy Sets Syst 85:305-309

Wang X-Z, Zhai J-H, Lu S-X (2008) Induction of multiple fuzzy decision trees based on rough set technique. Inf Sci 178:3188-3202

Wang X, Liu X, Zhang L (2014) A rapid fuzzy rule clustering method based on granular computing. Appl Soft Comput 24:534-542

Watada J, Tanaka H, Asai K (1986) Fuzzy discriminant analysis in fuzzy groups. Fuzzy Sets Syst 19:261-271

Wu HC (2003) Fuzzy least squares estimators in linear regression analysis for imprecise input and output data. Comput Statist Data Anal 42:203-217

Wu Q (2010) Regression application based on fuzzy $v$-support vector machine in symmetric triangular fuzzy space. Expert Systems Appl 37:2808-2814

Wu Q, Law R (2010) Fuzzy support vector regression machine with penalizing Gaussian noises on triangular fuzzy number space. Expert Systems Appl 37:7788-7795

Xian S, Qiu D, Zhang S (2013) A fuzzy principal component analysis approach to hierarchical evaluation model for balanced supply chain scorecard grading. J Optim Theory Appl 159:518-535

Xie X, Beni G (1991) A validity measure for fuzzy clustering. IEEE Trans Pattern Analysis Machine Intelligence 13(8):841-847

Yabuuch Y, Watada J (1997) Fuzzy principal component analysis and its application. Biomed Fuzzy Hum Sci 3:83-92

Yamashita T (2006) Fuzzy ratings and crisp feedback in fuzzy AHP for supporting human decision making. J Adv Comput Intel Intel Inf 10:219-224

Yang M, Ko C (1996) On a class of fuzzy c-numbers clustering procedures for fuzzy data. Fuzzy Sets Syst 84:49-60

Yang M, Liu H (1999) Fuzzy clustering procedures for conical fuzzy vector data. Fuzzy Sets Syst 106:189-200

Yang T-N, Wang S-D (2000) Fuzzy auto-associative neural networks for principal component extraction of noisy data. IEEE Trans Neural Netw 11:808-810

Yang MS, Wu KL (2004) A similarity-based robust clustering method. IEEE Trans Pattern Anal Mach Intell 26(4):434-448 
Yang MS, Wu K-L (2006) Unsupervised possibilistic clustering. Pattern Recognit 39:5-21

Yang M, Hwang P, Chen D (2004) Fuzzy clustering algorithms for mixed feature variables. Fuzzy Sets Syst 141:301-317

Yang C, Lu L, Lin H, Guan R, Shi X, Liang Y (2008) A fuzzy-statistics-based principal component analysis (FS-PCA) method for multispectral image enhancement and display. IEEE Trans Geosci Remote Sens 46:3937-3947

Yang M, Hung W, Chen D (2012) Self-organizing map for symbolic data. Fuzzy Sets Syst 203:49-73

Zadeh LA (1965) Fuzzy sets. Inf Control 8:338-353

Zadeh LA (1973) Outline of a new approach to the analysis of complex system and decision processes. IEEE Trans Syst Man Cyber 3:28-44

Zadeh LA (1975a) The concept of a linguistic variable and its application to approximate reasoning, I. Inform Sci 8:199-249

Zadeh LA (1975b) The concept of a linguistic variable and its application to approximate reasoning, II. Inform Sci 8:199-249

Zadeh LA (1975c) The concept of a linguistic variable and its application to approximate reasoning, III. Inf Sci 8:199-249
Zadeh L (1997) Information granulation and its centrality in human and machine intelligence. In: Grahne G. (ed) Proceedings of the 6. Scandinavian conference on artificial intelligence (SCAI'97). Frontiers in artificial intelligence and applications, 40. IOS Press, Amsterdam, pp 26-27

Zadeh L (2005) Toward a generalized theory of uncertainty (GTU) an outline. Inform Sci 172:1-40

Zarandi MH, Razaee ZS (2011) A fuzzy clustering model for fuzzy data with outliers. Int J Fuzzy Syst Appl (IJFSA) 1(2):29-42

Zeinalkani M, Eftekhari M (2014) Fuzzy partitioning of continuous attributes through discretization methods to constrict fuzzy decision tree classifiers. Inf Sci 278:715-735

Zimmermann HJ (2001) Fuzzy set theory and its applications. Kluwer Academic Press, Dordrecht

Zwick R, Carlstein E, Budescu D (1987) Measures of similarity among fuzzy concepts: A comparative analysis. Int $\mathbf{J}$ Approx Reason 1:221-242 